\title{
ALLELOPATHIC POTENTIAL OF PINUS ROXBURGHII NEEDLES AGAINST SELECTED WEEDS OF WHEAT CROP
}

\author{
ANWAR, T. ${ }^{1}$ - ILYAS, N. ${ }^{1}$ - QURESHI, R. ${ }^{1 *}$ MUNAZIR, M. ${ }^{2}$ - RAHIM, B. Z. ${ }^{3}$ - QURESHI, H. ${ }^{1}-$ \\ KousAR, R. ${ }^{1}$ - MAQSOOD, M. ${ }^{1}-$ ABBAS, Q. ${ }^{4}$ - BHATTI, M. I. ${ }^{5}$ - PANNI, M. K. ${ }^{6}$ \\ ${ }^{1}$ Department of Botany, Pir Mehr Ali Shah Arid Agriculture University \\ Murree Road, Shamsabad, 46300 Rawalpindi, Punjab, Pakistan \\ ${ }^{2}$ Department of Botany, Government College, Women University \\ 51040 Sialkot, Punjab, Pakistan \\ ${ }^{3}$ Department of Botany, Baluchistan University, 87500 Quetta, Baluchistan, Pakistan \\ ${ }^{4}$ Department of Biological Sciences, Karakorum International University \\ Gilgit-Baltistan, Gilgit, Pakistan \\ ${ }^{5}$ Department of Plant Pathology, Sindh Agriculture University \\ Sub-Campus Umarkot, Sindh, Pakistan \\ ${ }^{6}$ Institute of Biotechnology and Genetic Engineering (IBGE), The University of Agriculture \\ 25130 Peshawar, Pakistan \\ *Corresponding author \\ e-mail: rahmatullahq@yahoo.com \\ (Received $13^{\text {th }}$ Sep 2018; accepted $5^{\text {th }}$ Dec 2018)
}

\begin{abstract}
This study was aimed at evaluating allelopathic activity of various solvent extracts of Pinus roxburghii (Pine tree) against some important weeds viz. Phalaris minor (bunchgrass), Avena fatua (wild oat), Chenopodium album (pigweed), Euphorbia helioscopia (sun spurge) and Rumex dentatus (toothed dock) of bread wheat (Triticum aestivum) by employing sandwich method (powdered needles) on filter paper, soil and agar. The data attained from statistical analysis revealed that methanolic needle extract possessed the highest germination percentage inhibition for T. aestivum, followed by $C$. album and A. fatua applied in soil. Similarly, maximum radical length suppression was observed for $R$. dentatus, followed by $C$. album. The plumule length retardation was noted best in $R$. dentatus, followed by $A$. fatua. The application of methanolic $P$. roxburghii extract was responsible for reduction in seed germination, radical and plumule length of wheat. Based on results, it can be concluded that methanolic Pinus needles extract possesses potential inhibitory effects that required for further detailed analysis to establish allelopathic potential and onward application to be used as phytoherbicide.
\end{abstract}

Keywords: Allelopathic potential, Bread wheat, Pinus needles, Growth retardation, Methanol extract, Weed management

\section{Introduction}

Allelopathy is the negative effects of one plant on the germination, growth, and/or development of other plants. The chemicals which execute allelopathy are commonly known as allelochemicals. Plant release chemical into the environment and these chemicals affect physiological functions of other plants in immediate vicinity, such as seed germination, photosynthesis, respiration, transpiration, stomatal behavior and ion uptake (Anwar et al., 2017a). Allelochemicals come from the class of secondary metabolites that are produced as by-products in the primary metabolic pathways of the 
plants. Like many other natural compounds, these chemicals have the capacity to cause a wide array of biological effects and can be quite useful for agriculture systems as well as weed control practices (Anwar et al., 2013). Allelochemicals include alkaloids, flavonoids phenoloids, and glucosionates. These are produced by the plants during their growth and developmental processes (Ahmed et al., 2014). Allelochemicals have been found to act as agent in the formation and disintegration of plant hormones, for instance they play role in the activation of Abscisic acid (ABA) synthesis via the action of ferulic acid (Zafar et al., 2010; Zhou et al., 2004).

Pinus roxburghii Sarg. (Family: Pinaceae) commonly known as "chir pine" is native to Himalaya, the region extends from northern Pakistan, across northern India and Nepal to Bhutan. Dried needles of Pine trees forms a dense carpet on the forest floor, which are gathered by the locals in large bundles to serve as bedding for their cattle, for the year round. Still a large quantity of these needles is left on the forest floor and with the rain water these needles are weathered and the leachates from them are mixed with the surrounding soil environment. Needles and bark of $P$. roxburghii is reported to possess secondary metabolites such as alkaloids, glycosides, flavonoids, saponins, tannins and triterpines which may have potential aliphatic actions (Baroniya and Baroniya, 2014). It is concept that Wheat in the high mountain areas, do not reach to maturity in most cases and as such the crop is harvested premature and used as fodder. However, past temperature trends in the high mountain areas (Chitral district) have led to shortening of the growing season length which certainly has helped in increasing wheat yield as well as crop area in high mountain areas (Hussain et al., 2005). In this context, a study was carried out to evaluate allelopathic activity of Pinus roxburghii (Pine tree) against some important weeds viz. Phalaris minor (bunchgrass), Avena fatua (wild oat), Chenopodium album (pigweed), Euphorbia helioscopia (sun spurge) and Rumex dentatus (toothed dock) of wheat crop.

\section{Materials and methods}

\section{Collection and mechanical processing of $P$. roxburghii needles}

Mature needles (500 g) of P. roxburghii were collected from district Rawalpindi $\left(73^{\circ}\right.$ 02' E longitude and 33 $36^{\circ} \mathrm{N}$ latitude, $508 \mathrm{~m}$ above sea level) Punjab, Pakistan during November, 2017 and washed for several times under running tap water. The needles were dried for 4 weeks in shade at $30^{\circ} \mathrm{C}$. The dried needles were crushed using heavy duty blender to make fine powder by passing through mesh size $2 \mathrm{~mm}$ and kept in air tight plastic zip lock bags at $4{ }^{\circ} \mathrm{C}$ (Anwar et al., 2013).

\section{Procurement and surface sterilization of test species seeds}

Seeds of major weeds of T. aestivum viz. P. minor, A. fatua, C. album, E.helioscopia and $R$. dentatus were procured from Barani Agricultural Research Institute (BARI), Chakwal, District Rawalpindi, Punjab, Pakistan. Seeds (15g) of each test species were surface sterilized with $2 \%(\mathrm{w} / \mathrm{v})$ solution of Sodium hypochlorite $(\mathrm{NaOCl})$ for 1-2 min. After disinfection seeds were washed with distilled water (Anwar et al., 2016; Biljana and Kragujevac, 2015). 


\section{Filter paper and soil bioassay with P. roxburghii needles solvent extracts}

Dried needles powder was extracted in distilled water, hexane and methanol separately at $30^{\circ} \mathrm{C}$ for $24 \mathrm{~h}$ on an orbital shaker (160rpm). The extract was filtered through Whatman filter paper No.1. The stock solution was diluted to prepare different concentration i.e. $\mathrm{T}_{1}(100 \%), \mathrm{T}_{2}(75 \%), \mathrm{T}_{3}(50 \%)$ and $\mathrm{T}_{4}$ (control) (Sahu and Devkota, 2013; Anwar et al., 2017a).

An aliquot of $15 \mathrm{ml}$ extract was added on $25 \mathrm{~g}$ soil and $5 \mathrm{ml}$ extract was added on filter paper per Petri dish. Distilled water, blank hexane and methanol was used as control in respective solvent extract bioassay. Ten seeds of selected test species were used per Petri dish. Each treatment was replicated for three times. The Petri dishes were wrapped with aluminium foil and incubated in growth chamber (NTS Model MI$25 \mathrm{~S}$ ) at $28^{\circ} \mathrm{C}$ for 15 days. The germination percentages, lengths of radical and plumule were calculated for each test species by comparing with respective control (Khan et al., 2008).

\section{Filter paper and soil bioassay with P. roxburghii needles dried powder}

Dried powder (10mg) of $P$. roxburghii needles was added on filter paper along with $5 \mathrm{ml}$ distilled water per Petri dish. Similarly, 50mg powder was added on $25 \mathrm{~g}$ soil along with $15 \mathrm{ml}$ distilled water per Petri dish (Raana et al., 2012). Ten surface sterilized seeds of each test species were placed in sterilized Petri dishes. The Petri dishes were wrapped with aluminium foil and incubated in growth chamber (NTS Model MI-25S) at $25^{\circ} \mathrm{C}$ for 15 days. The germination percentages, lengths of radical and plumule were calculated for each test species (Anwar et al., 2017b).

\section{Sandwich method}

Five $\mathrm{ml}$ of $0.75 \%(\mathrm{w} / \mathrm{v})$ agar (Nalge Nunc Intl., Roskilde, Denmark) was poured in each of the six-well $\left(10 \mathrm{~cm}^{2}\right.$ area/well) into multi-dish plastic plate. The agar solution was left for solidification. The powder of $P$. roxburghii needles was placed @ 10 and 50 $\mathrm{mg}$ in wells of plate and roofed by a thin layer of $0.75 \%$ (w/v) agar. After solidification, 10 seeds of each test species were placed on agar gel in each well of the plate (Fujii et al., 2003, 2004). The multi-well plastic plates were incubated in growth chamber (NTS Model MI-25S) at $25^{\circ} \mathrm{C}$ for 15 days. Each treatment was replicated three times. The germination percentages, lengths of radical and plumule for each test species were calculated.

A completely randomized design (CRD) was used for experiment analysis. The statistical analysis was done using STATISTIX 9. Means were separated by Fisher's protected LSD test (Nekonam et al., 2014).

\section{Results}

\section{Allelopathic potential of $P$. roxburghii aqueous extract}

\section{Germination percentage}

The results revealed that aqueous extract inhibited 54\%, $48 \%$ and $43 \%$ germination percentage of T. aestivum, C. album and A. fatua, respectively on filter paper (Table 1), whereas, no significant inhibitory effect on the germination of $R$. dentatus, $P$. minor and $E$. helioscopia was observed supposed to be resistance against $P$. roxburghii extract. 
Similarly, $P$. roxburghii aqueous extract on soil significantly inhibited $59 \%, 50 \%$ and $44 \%$ seed germination of $T$. aestivum, $C$. album and A. fatua, respectively compared to the control (Table 2). It has been observed that maximum (98\%) germination was observed for $R$. dentatus, $P$. minor and E. helioscopia. During the experimentation, minimum germination was noted for T. aestivum (i.e. $46 \%$ and $41 \%$ ) on filter paper and soil, respectively. The results revealed that germination percentage reduction of the $T$. aestivum, $C$. album and A. fatua were concentration dependent, with the increase of concentration, the suppression potential was gradually enhanced (Fig. Ia).

\section{Radical length}

The aqueous extract of $P$. roxburghii exhibited radical length inhibition of $C$. album (40\%) followed by $R$. dentatus (39\%) on filter paper (Table 3), whereas, no significant effect was noted for $T$. aestivum, P. minor, E. helioscopia and A. fatua showing resistance against extract. Similarly, the applications of extract into soil significantly suppressed radical length of $C$. album and $R$. dentatus with $46 \%$ and $41 \%$ respectively as compared to control (Table 4). The maximum (98\%) radical length was observed for T. aestivum, $P$. minor, E. helioscopia and A. fatua. The final data concluded that minimum radical length was noted for $C$. album i.e. $60 \%$ and $64 \%$ on filter paper and soil, respectively (Fig. 1b).

Table 1. Allelopathic effect of $P$. roxburghii aqueous extract (AE) on germination percentage of test species on filter paper

\begin{tabular}{c|c|c|c|c|c|c}
\hline \multirow{2}{*}{ Treatments } & \multicolumn{6}{|c}{ Test species } \\
\cline { 2 - 6 } & T. aestivum & A. fatua & R. dentatus & P. minor & E. helioscopia & C. album \\
\hline $\mathrm{T}_{1 \mathrm{AE}}$ & $42^{\mathrm{d}}$ & $51^{\mathrm{d}}$ & $80^{\mathrm{a}}$ & $82^{\mathrm{a}}$ & $84^{\mathrm{a}}$ & $47^{\mathrm{d}}$ \\
$\mathrm{T}_{2} \mathrm{AE}$ & $66^{\mathrm{c}}$ & $65^{\mathrm{c}}$ & $82^{\mathrm{a}}$ & $84^{\mathrm{a}}$ & $86^{\mathrm{a}}$ & $68^{\mathrm{c}}$ \\
$\mathrm{T}_{3 \mathrm{AE}}$ & $79^{\mathrm{b}}$ & $78^{\mathrm{b}}$ & $83^{\mathrm{a}}$ & $85^{\mathrm{a}}$ & $87^{\mathrm{a}}$ & $79^{\mathrm{b}}$ \\
$\mathrm{T}_{4} \mathrm{AE}$ & $91^{\mathrm{a}}$ & $89^{\mathrm{a}}$ & $84^{\mathrm{a}}$ & $86^{\mathrm{a}}$ & $88^{\mathrm{a}}$ & $91^{\mathrm{a}}$ \\
${ }^{1} \mathrm{LSD}$ & 12.554 & 18.510 & 14.844 & 13.08 & 15.580 & 15.541 \\
${ }^{2} \mathrm{~F}-\mathrm{value}$ & $14.63^{*}$ & $21.36^{*}$ & $23.04^{*}$ & $39.81^{*}$ & $52.38^{*}$ & $44.98^{*}$ \\
\hline
\end{tabular}

Means followed by different letters within one column differ significantly at $P<5 \%$

*Significant at $P<1 \%$

Table 2. Allelopathic effect of $P$. roxburghii aqueous extract (AE) on germination percentage of test species on soil

\begin{tabular}{c|c|c|c|c|c|c}
\hline \multirow{2}{*}{ Treatments } & \multicolumn{6}{|c}{ Test species } \\
\cline { 2 - 7 } & T. aestivum & A. fatua & R. dentatus & P. minor & E. helioscopia & C. album \\
\hline $\mathrm{T}_{1 \mathrm{AE}}$ & $38^{\mathrm{d}}$ & $50^{\mathrm{c}}$ & $81^{\mathrm{a}}$ & $83^{\mathrm{a}}$ & $85^{\mathrm{a}}$ & $46^{\mathrm{d}}$ \\
$\mathrm{T}_{2 \mathrm{AE}}$ & $55^{\mathrm{c}}$ & $53^{\mathrm{c}}$ & $83^{\mathrm{a}}$ & $85^{\mathrm{a}}$ & $87^{\mathrm{a}}$ & $56^{\mathrm{c}}$ \\
$\mathrm{T}_{3 \mathrm{AE}}$ & $70^{\mathrm{b}}$ & $72^{\mathrm{b}}$ & $84^{\mathrm{a}}$ & $86^{\mathrm{a}}$ & $88^{\mathrm{a}}$ & $72^{\mathrm{b}}$ \\
$\mathrm{T}_{4 \mathrm{AE}}$ & $92^{\mathrm{a}}$ & $90^{\mathrm{a}}$ & $85^{\mathrm{a}}$ & $87^{\mathrm{a}}$ & $89^{\mathrm{a}}$ & $92^{\mathrm{a}}$ \\
${ }^{1} \mathrm{LSD}$ & 19.808 & 18.990 & 16.60 & 16.435 & 18.67 & 22.33 \\
${ }^{2} \mathrm{~F}-\mathrm{value}$ & $10.99^{* *}$ & $18.19^{*}$ & $34.19^{*}$ & $22.97^{*}$ & $31.71^{*}$ & $18.61^{*}$ \\
\hline
\end{tabular}

Means followed by different letters within one column differ significantly at $P<5 \%$

*Significant at $P<1 \%$ 
Table 3. Allelopathic effect of $P$. roxburghii aqueous extract $(A E)$ on radical length ( $\mathrm{cm})$ of test species on filter paper

\begin{tabular}{c|c|c|c|c|c|c}
\hline \multirow{2}{*}{ Treatments } & \multicolumn{6}{|c}{ Test species } \\
\cline { 2 - 7 } & T. aestivum & A. fatua & R. dentatus & P. minor & E. helioscopia & C. album \\
\hline $\mathrm{T}_{1 \mathrm{AE}}$ & $8.15^{\mathrm{a}}$ & $7.11^{\mathrm{a}}$ & $5.59^{\mathrm{d}}$ & $8.12^{\mathrm{a}}$ & $7.16^{\mathrm{a}}$ & $5.52^{\mathrm{c}}$ \\
$\mathrm{T}_{2} \mathrm{AE}$ & $8.24^{\mathrm{a}}$ & $7.18^{\mathrm{a}}$ & $6.76^{\mathrm{c}}$ & $8.25^{\mathrm{a}}$ & $7.23^{\mathrm{a}}$ & $6.37^{\mathrm{c}}$ \\
$\mathrm{T}_{3 \mathrm{AE}}$ & $8.31^{\mathrm{a}}$ & $7.21^{\mathrm{a}}$ & $7.99^{\mathrm{b}}$ & $8.34^{\mathrm{a}}$ & $7.34^{\mathrm{a}}$ & $7.81^{\mathrm{b}}$ \\
$\mathrm{T}_{4 \mathrm{AE}}$ & $8.39^{\mathrm{a}}$ & $7.22^{\mathrm{a}}$ & $9.12^{\mathrm{a}}$ & $8.39^{\mathrm{a}}$ & $7.46^{\mathrm{a}}$ & $9.17^{\mathrm{a}}$ \\
${ }^{1} \mathrm{LSD}$ & 1.640 & 0.899 & 1.2125 & 1.1662 & 1.420 & 0.8677 \\
${ }^{2} \mathrm{~F}-\mathrm{value}$ & $46.47^{*}$ & $65.33^{*}$ & $112.84^{*}$ & $95.02^{*}$ & $55.72^{*}$ & $134.19^{*}$ \\
\hline
\end{tabular}

Means followed by different letters within one column differ significantly at $P<5 \%$

*Significant at $P<1 \%$

Table 4. Allelopathic effect of $P$. roxburghii aqueous extract $(A E)$ on radical length $(\mathrm{cm})$ of test species on soil

\begin{tabular}{c|c|c|c|c|c|c}
\hline \multirow{2}{*}{ Treatments } & \multicolumn{6}{|c}{ Test species } \\
\cline { 2 - 7 } & T. aestivum & A. fatua & R. dentatus & P. minor & E. helioscopia & C. album \\
\hline $\mathrm{T}_{1 \mathrm{AE}}$ & $8.34^{\mathrm{a}}$ & $7.21^{\mathrm{a}}$ & $5.46^{\mathrm{c}}$ & $8.46^{\mathrm{a}}$ & $7.35^{\mathrm{a}}$ & $5.11^{\mathrm{d}}$ \\
$\mathrm{T}_{2 \mathrm{AE}}$ & $8.42^{\mathrm{a}}$ & $7.39^{\mathrm{a}}$ & $6.28^{\mathrm{c}}$ & $8.57^{\mathrm{a}}$ & $7.45^{\mathrm{a}}$ & $6.15^{\mathrm{c}}$ \\
$\mathrm{T}_{3 \mathrm{AE}}$ & $8.51^{\mathrm{a}}$ & $7.43^{\mathrm{a}}$ & $7.41^{\mathrm{b}}$ & $8.62^{\mathrm{a}}$ & $7.57^{\mathrm{a}}$ & $7.41^{\mathrm{b}}$ \\
$\mathrm{T}_{4 \mathrm{AE}}$ & $8.61^{\mathrm{a}}$ & $7.45^{\mathrm{a}}$ & $9.34^{\mathrm{a}}$ & $8.65^{\mathrm{a}}$ & $7.68^{\mathrm{a}}$ & $9.38^{\mathrm{a}}$ \\
${ }^{1} \mathrm{LSD}$ & 1.1027 & 2.7087 & 1.2058 & 0.9098 & 0.8741 & 0.7677 \\
${ }^{2} \mathrm{~F}-$ value & $93.48^{*}$ & $46.38^{*}$ & $98.75^{*}$ & $217.04^{*}$ & $162.39^{*}$ & $135.19^{*}$ \\
\hline
\end{tabular}

Means followed by different letters within one column differ significantly at $P<5 \%$

*Significant at $P<1 \%$

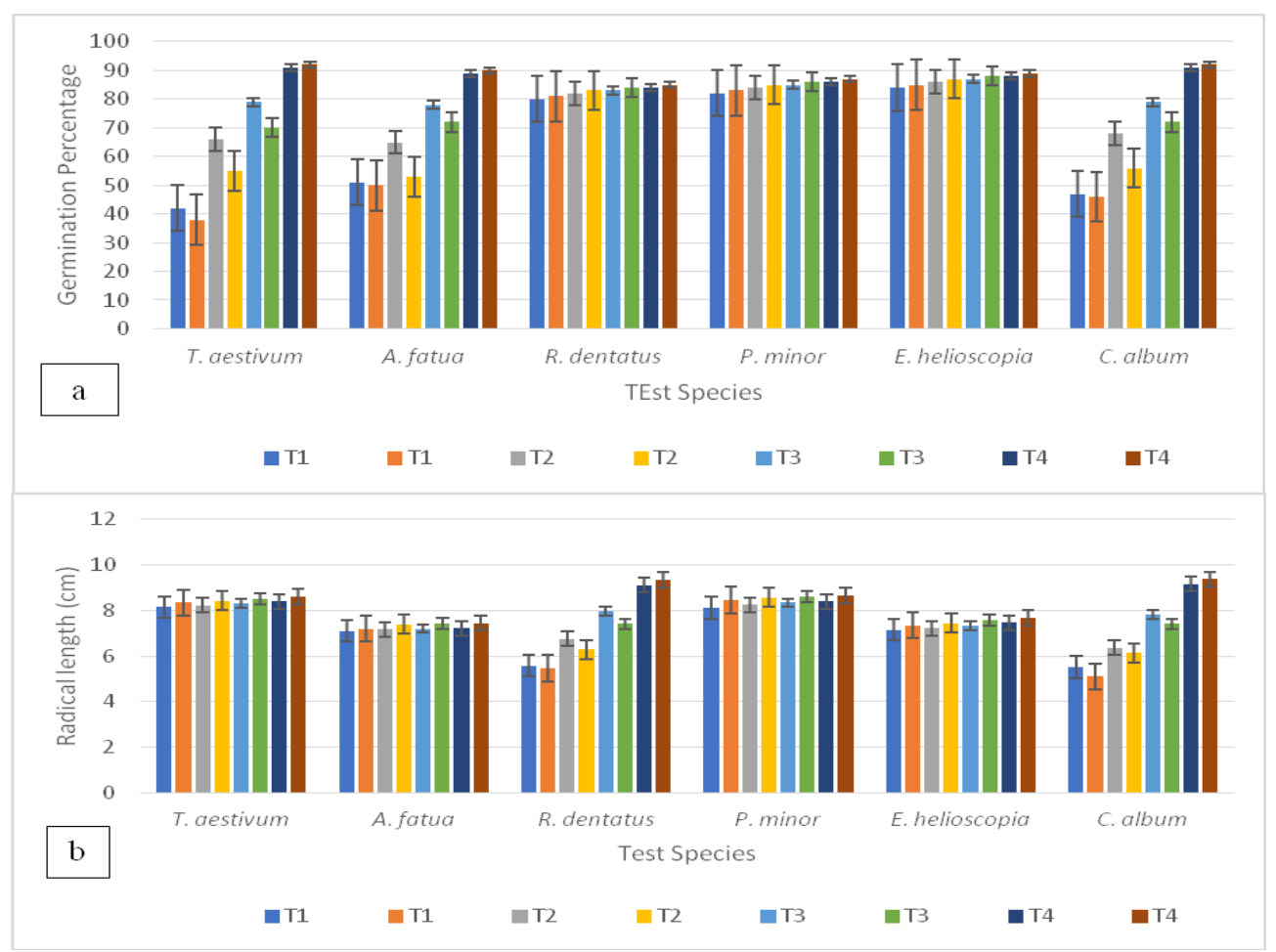




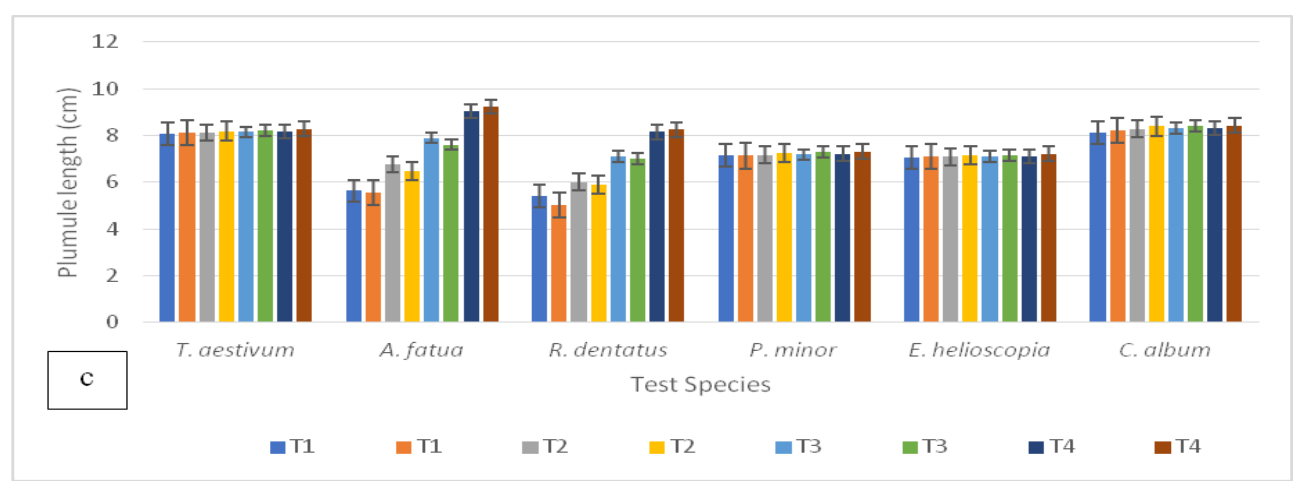

Figure 1. Allelopathic potential of $P$. roxburghii aqueous extract against test species on filter paper $(F P)$ and soil $(S)$ on: (a) germination percentage $(b)$ radical length $(c)$ plumule length $\left(T_{1}\right.$ $=100 \%, T_{2}=75 \%, T_{3}=50 \%$ and $T_{4}=$ control $)$

\section{Plumule length}

The aqueous extract of $P$. roxburghii significantly inhibited the plumule length of A. fatua (38\%) and R. dentatus (34\%) as compared control on filter paper (Table $5)$. Remarkably, there was no momentous effect on plumule elongation of $T$. aestivum, $P$. minor, E. helioscopia and $C$. album. Likewise, $P$. roxburghii aqueous extract significantly inhibited plumule length of $A$. fatua (40\%) and $R$. dentatus $(39 \%)$ as compared to control in soil (Table 6). The statistical figures also proposed that maximum plumule length (98\%) was noted for $T$. aestivum, $P$. minor, E. helioscopia and C. album. The statistical data concluded that minimum plumule length was noted for A. fatua showing $62 \%$ and $60 \%$ on filter paper and soil, respectively (Fig. 1c).

Table 5. Allelopathic effect of $P$. roxburghii aqueous extract $(A E)$ on plumule length ( $\mathrm{cm}$ ) of test species on filter paper

\begin{tabular}{c|c|c|c|c|c|c}
\hline \multirow{2}{*}{ Treatments } & \multicolumn{6}{|c}{ Test species } \\
\cline { 2 - 7 } & T. aestivum & A. fatua & R. dentatus & P. minor & E. helioscopia & C. album \\
\hline $\mathrm{T}_{1 \mathrm{AE}}$ & $8.08^{\mathrm{a}}$ & $5.63^{\mathrm{d}}$ & $5.41^{\mathrm{c}}$ & $7.14^{\mathrm{a}}$ & $7.06^{\mathrm{a}}$ & $8.12^{\mathrm{a}}$ \\
$\mathrm{T}_{2 \mathrm{AE}}$ & $8.11^{\mathrm{a}}$ & $6.78^{\mathrm{c}}$ & $6.01^{\mathrm{c}}$ & $7.17^{\mathrm{a}}$ & $7.08^{\mathrm{a}}$ & $8.29^{\mathrm{a}}$ \\
$\mathrm{T}_{3 \mathrm{AE}}$ & $8.15^{\mathrm{a}}$ & $7.89^{\mathrm{b}}$ & $7.11^{\mathrm{b}}$ & $7.19^{\mathrm{a}}$ & $7.10^{\mathrm{a}}$ & $8.31^{\mathrm{a}}$ \\
$\mathrm{T}_{4 \mathrm{AE}}$ & $8.18^{\mathrm{a}}$ & $9.04^{\mathrm{a}}$ & $8.15^{\mathrm{a}}$ & $7.22^{\mathrm{a}}$ & $7.11^{\mathrm{a}}$ & $8.33^{\mathrm{a}}$ \\
${ }^{1} \mathrm{LSD}$ & 2.3332 & 1.4291 & 0.8679 & 1.2428 & 0.7319 & 0.8297 \\
${ }^{2} \mathrm{~F}-\mathrm{value}$ & $27.95^{*}$ & $23.83^{*}$ & $81.03^{*}$ & $29.85^{*}$ & $219.87^{*}$ & $222.41^{*}$ \\
\hline
\end{tabular}

Means followed by different letters within one column differ significantly at $P<5 \%$

* Significant at $P<1 \%$ 
Table 6. Allelopathic effect of $P$. roxburghii aqueous extract $(A E)$ on plumule length $(\mathrm{cm})$ of test species on soil

\begin{tabular}{c|c|c|c|c|c|c}
\hline \multirow{2}{*}{ Treatments } & \multicolumn{6}{|c}{ Test species } \\
\cline { 2 - 7 } & T. aestivum & A. fatua & R. dentatus & P. minor & E. helioscopia & C. album \\
\hline $\mathrm{T}_{1 \mathrm{AE}}$ & $8.13^{\mathrm{a}}$ & $5.54^{\mathrm{c}}$ & $5.01^{\mathrm{c}}$ & $7.13^{\mathrm{a}}$ & $7.12^{\mathrm{a}}$ & $8.22^{\mathrm{a}}$ \\
$\mathrm{T}_{2 \mathrm{AE}}$ & $8.19^{\mathrm{a}}$ & $6.48^{\mathrm{c}}$ & $5.89^{\mathrm{c}}$ & $7.26^{\mathrm{a}}$ & $7.14^{\mathrm{a}}$ & $8.39^{\mathrm{a}}$ \\
$\mathrm{T}_{3 \mathrm{AE}}$ & $8.23^{\mathrm{a}}$ & $7.61^{\mathrm{b}}$ & $6.99^{\mathrm{b}}$ & $7.31^{\mathrm{a}}$ & $7.17^{\mathrm{a}}$ & $8.41^{\mathrm{a}}$ \\
$\mathrm{T}_{4 \mathrm{AE}}$ & $8.28^{\mathrm{a}}$ & $9.24^{\mathrm{a}}$ & $8.25^{\mathrm{a}}$ & $7.32^{\mathrm{a}}$ & $7.21^{\mathrm{a}}$ & $8.43^{\mathrm{a}}$ \\
${ }^{1} \mathrm{LSD}$ & 2.4502 & 0.9978 & 0.7720 & 1.1434 & 0.8468 & 1.1019 \\
${ }^{2} \mathrm{~F}-\mathrm{value}$ & $9.54^{* *}$ & $72.85^{*}$ & $102.44^{*}$ & $72.9^{*}$ & $36.04^{*}$ & $86.41^{*}$ \\
\hline
\end{tabular}

Means followed by different letters within one column differ significantly at $P<5 \%$

*Significant at $P<1 \%$

\section{Allelopathic potential of $P$. roxburghii hexane extract}

\section{Germination percentage}

It has been observed from the results that there was significant reduction of germination of $T$. aestivum, C. album and A. fatua showing 54\%, $48 \%$ and $43 \%$ respectively as compared to their respective control on filter paper (Table 7), whereas, no significant effect on the germination of $R$. dentatus, $P$. minor and E. helioscopia, showing resistance to the allelopathic $P$. roxburghii hexane extract. Similarly, $P$. roxburghii hexane extract applied into soil showed the highest degree of inhibition of seed germination of T. aestivum, C. album and A. fatua with $62 \%, 52 \%$ and $49 \%$ respectively as compared to their respective control (Table 8). The maximum (98\%) germination was noted for $R$. dentatus, $P$. minor and E. helioscopia. In the present study it was noted that minimum germination was noted for T. aestivum i.e. $46 \%$ and $38 \%$ on filter paper and soil. The statistics also recommended that allelopathic inhibitory effect was concentration dependent for T. aestivum, C. album and A. fatua with concentration increase, suppression potential was gradually enhanced (Fig. 2a).

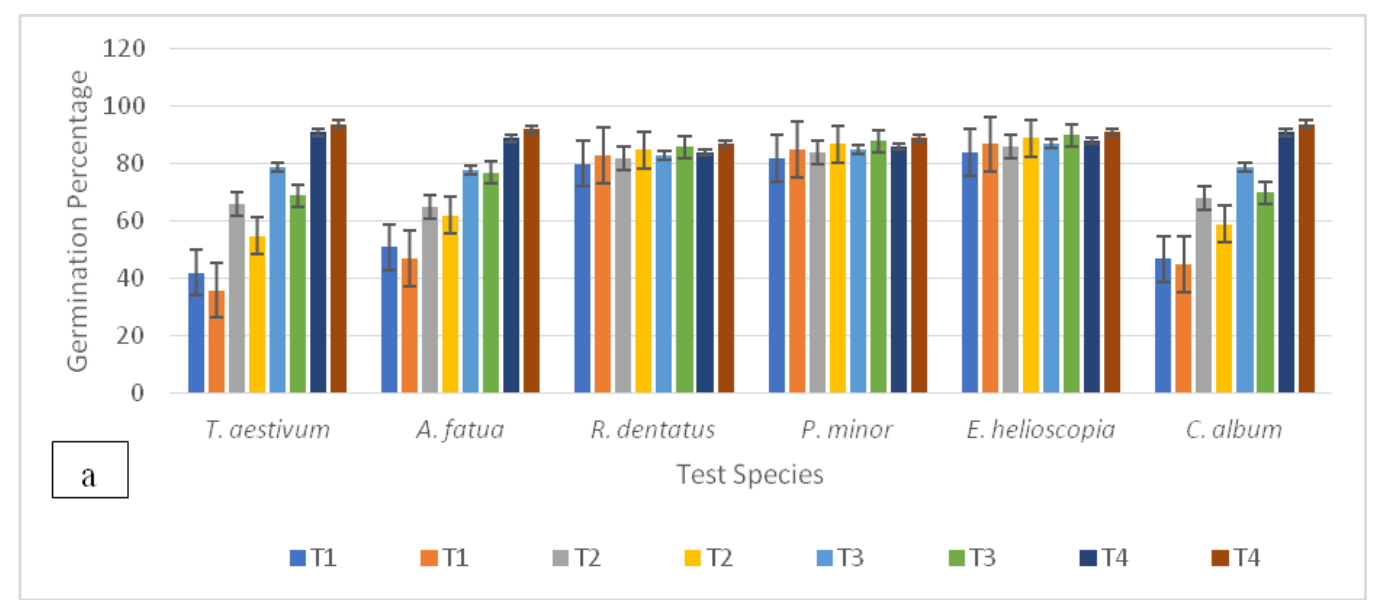




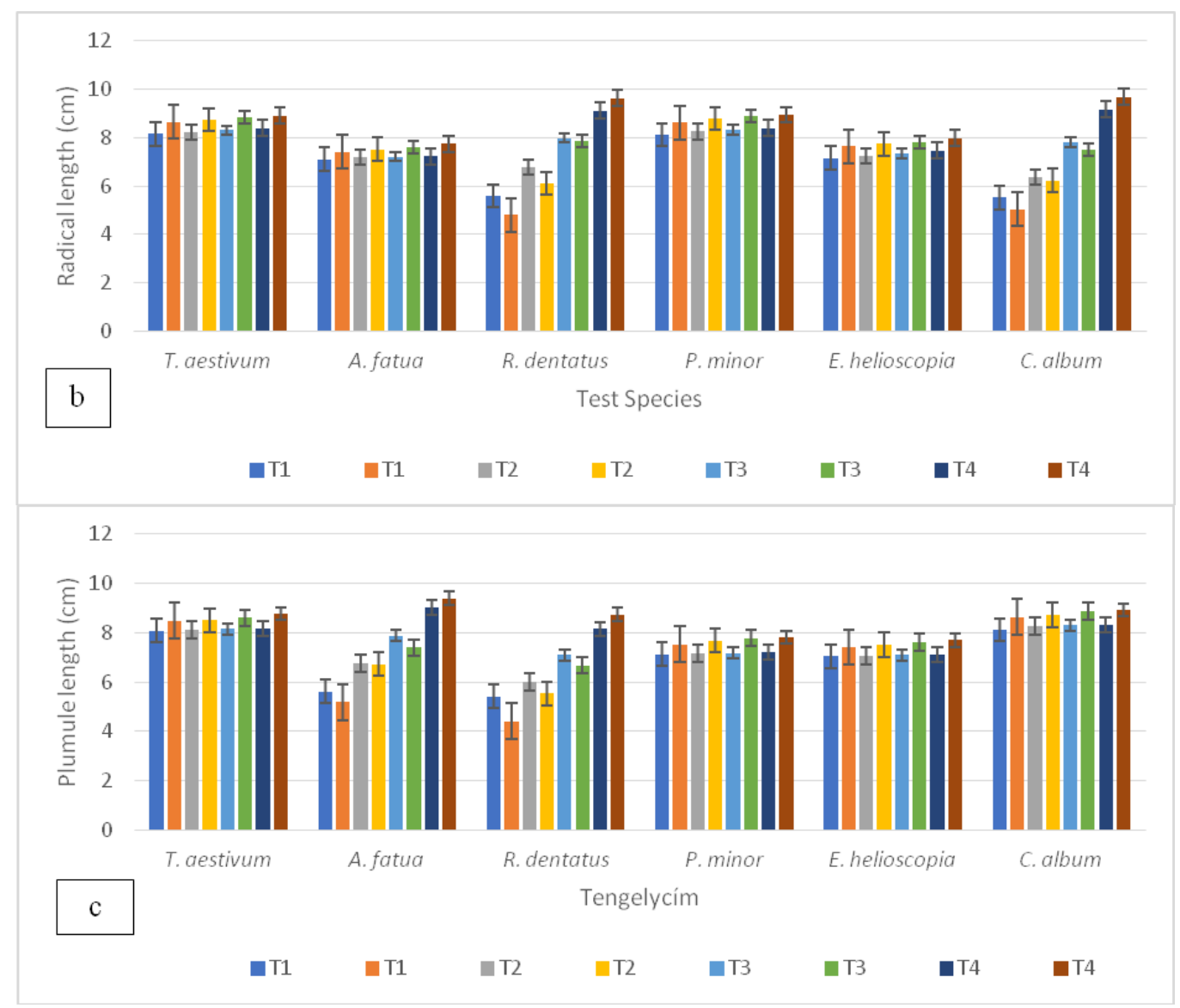

Figure 2. Allelopathic potential of $P$. roxburghii hexane extract against test species on filter paper $(F P)$ and soil $(S)$ on: (a) germination percentage $(b)$ radical length $(c)$ plumule length $\left(T_{1}\right.$ $=100 \%, T_{2}=75 \%, T_{3}=50 \%$ and $T_{4}=$ control $)$

Table 7. Allelopathic effect of $P$. roxburghii hexane extract (HE) on germination percentage of test species on filter paper

\begin{tabular}{c|c|c|c|c|c|c}
\hline \multirow{2}{*}{ Treatments } & \multicolumn{6}{|c}{ Test species } \\
\cline { 2 - 7 } & T. aestivum & A. fatua & R. dentatus & P. minor & E. helioscopia & C. album \\
\hline $\mathrm{T}_{1 \mathrm{HE}}$ & $42^{\mathrm{d}}$ & $51^{\mathrm{d}}$ & $80^{\mathrm{a}}$ & $82^{\mathrm{a}}$ & $84^{\mathrm{a}}$ & $47^{\mathrm{d}}$ \\
$\mathrm{T}_{2 \mathrm{HE}}$ & $66^{\mathrm{c}}$ & $65^{\mathrm{c}}$ & $82^{\mathrm{a}}$ & $84^{\mathrm{a}}$ & $86^{\mathrm{a}}$ & $68^{\mathrm{c}}$ \\
$\mathrm{T}_{3 \mathrm{HE}}$ & $79^{\mathrm{b}}$ & $78^{\mathrm{b}}$ & $83^{\mathrm{a}}$ & $85^{\mathrm{a}}$ & $87^{\mathrm{a}}$ & $79^{\mathrm{b}}$ \\
$\mathrm{T}_{4 \mathrm{HE}}$ & $91^{\mathrm{a}}$ & $89^{\mathrm{a}}$ & $84^{\mathrm{a}}$ & $86^{\mathrm{a}}$ & $88^{\mathrm{a}}$ & $91^{\mathrm{a}}$ \\
${ }^{1} \mathrm{LSD}$ & 12.554 & 18.510 & 14.844 & 13.08 & 15.580 & 15.541 \\
${ }^{2} \mathrm{~F}-\mathrm{value}$ & $14.63^{*}$ & $21.36^{*}$ & $23.04^{*}$ & $39.81^{*}$ & $52.38^{*}$ & $44.98^{*}$ \\
\hline
\end{tabular}

Means followed by different letters within one column differ significantly at $P<5 \%$

*Significant at $P<1 \%$ 
Table 8. Allelopathic effect of $P$. roxburghii hexane extract $(H E)$ on germination percentage of test species on soil

\begin{tabular}{c|c|c|c|c|c|c}
\hline \multirow{2}{*}{ Treatments } & \multicolumn{6}{|c}{ Test species } \\
\cline { 2 - 7 } & T. aestivum & A. fatua & R. dentatus & P. minor & E. helioscopia & C. album \\
\hline $\mathrm{T}_{1 \mathrm{HE}}$ & $36^{\mathrm{d}}$ & $47^{\mathrm{d}}$ & $83^{\mathrm{a}}$ & $85^{\mathrm{a}}$ & $87^{\mathrm{a}}$ & $45^{\mathrm{d}}$ \\
$\mathrm{T}_{2} \mathrm{HE}$ & $55^{\mathrm{c}}$ & $62^{\mathrm{c}}$ & $85^{\mathrm{a}}$ & $87^{\mathrm{a}}$ & $89^{\mathrm{a}}$ & $59^{\mathrm{c}}$ \\
$\mathrm{T}_{3} \mathrm{HE}$ & $69^{\mathrm{b}}$ & $77^{\mathrm{b}}$ & $86^{\mathrm{a}}$ & $88^{\mathrm{a}}$ & $90^{\mathrm{a}}$ & $70^{\mathrm{b}}$ \\
$\mathrm{T}_{4 \mathrm{HE}}$ & $94^{\mathrm{a}}$ & $92^{\mathrm{a}}$ & $87^{\mathrm{a}}$ & $89^{\mathrm{a}}$ & $91^{\mathrm{a}}$ & $94^{\mathrm{a}}$ \\
${ }^{1} \mathrm{LSD}$ & 13.722 & 18.26 & 18.774 & 13.74 & 14.529 & 17.77 \\
${ }^{2} \mathrm{~F}-\mathrm{value}$ & $25.64^{*}$ & $22.09^{*}$ & $15.39^{*}$ & $16.90^{*}$ & $20.73^{*}$ & $32.81^{*}$ \\
\hline
\end{tabular}

Means followed by different letters within one column differ significantly at $P<5 \%$

*Significant at $P<1 \%$

\section{Radical length}

It is also clear from the result that C. album (40\%) followed by R. dentatus (39\%) exhibited radical length inhibition in $P$. roxburghii hexane extract on filter paper (Table 9), whereas, no noteworthy effect on radical length of T. aestivum, P. minor, E. helioscopia and A. fatua showing resistance to extract. Likewise, $P$. roxburghii hexane extract on soil cause significant radical length reduction of $R$. dentatus (50\%) and $C$. album (48\%) as compared to control (Table 10).

Table 9. Allelopathic effect of $P$. roxburghii hexane extract $(H E)$ on radical length $(\mathrm{cm})$ of test species on filter paper

\begin{tabular}{c|c|c|c|c|c|c}
\hline \multirow{2}{*}{ Treatments } & \multicolumn{6}{|c}{ Test species } \\
\cline { 2 - 7 } & T. aestivum & A. fatua & R. dentatus & P. minor & E. helioscopia & C. album \\
\hline $\mathrm{T}_{1 \mathrm{HE}}$ & $8.15^{\mathrm{a}}$ & $7.11^{\mathrm{a}}$ & $5.59^{\mathrm{d}}$ & $8.12^{\mathrm{a}}$ & $7.16^{\mathrm{a}}$ & $5.52^{\mathrm{c}}$ \\
$\mathrm{T}_{2 \mathrm{HE}}$ & $8.24^{\mathrm{a}}$ & $7.18^{\mathrm{a}}$ & $6.76^{\mathrm{c}}$ & $8.25^{\mathrm{a}}$ & $7.23^{\mathrm{a}}$ & $6.37^{\mathrm{c}}$ \\
$\mathrm{T}_{3 \mathrm{HE}}$ & $8.31^{\mathrm{a}}$ & $7.21^{\mathrm{a}}$ & $7.99^{\mathrm{b}}$ & $8.34^{\mathrm{a}}$ & $7.34^{\mathrm{a}}$ & $7.81^{\mathrm{b}}$ \\
$\mathrm{T}_{4 \mathrm{HE}}$ & $8.39^{\mathrm{a}}$ & $7.22^{\mathrm{a}}$ & $9.12^{\mathrm{a}}$ & $8.39^{\mathrm{a}}$ & $7.46^{\mathrm{a}}$ & $9.17^{\mathrm{a}}$ \\
${ }^{1} \mathrm{LSD}$ & 1.640 & 0.899 & 1.2125 & 1.1662 & 1.420 & 0.8677 \\
${ }^{2} \mathrm{~F}-\mathrm{value}$ & $46.47^{*}$ & $65.33^{*}$ & $112.84^{*}$ & $95.02^{*}$ & $55.72^{*}$ & $134.19^{*}$ \\
\hline
\end{tabular}

Means followed by different letters within one column differ significantly at $P<5 \%$

*Significant at $P<1 \%$

Table 10. Allelopathic effect of $P$. roxburghii hexane extract $(H E)$ on radical length $(\mathrm{cm})$ of test species on soil

\begin{tabular}{c|c|c|c|c|c|c}
\hline \multirow{2}{*}{ Treatments } & \multicolumn{7}{|c}{ Test species } \\
\cline { 2 - 7 } & T. aestivum & A. fatua & R. dentatus & P. minor & E. helioscopia & C. album \\
\hline $\mathrm{T}_{1 \mathrm{HE}}$ & $8.66^{\mathrm{a}}$ & $7.42^{\mathrm{a}}$ & $4.79^{\mathrm{d}}$ & $8.63^{\mathrm{a}}$ & $7.65^{\mathrm{a}}$ & $5.04^{\mathrm{d}}$ \\
$\mathrm{T}_{2 \mathrm{HE}}$ & $8.73^{\mathrm{a}}$ & $7.52^{\mathrm{a}}$ & $6.11^{\mathrm{c}}$ & $8.78^{\mathrm{a}}$ & $7.74^{\mathrm{a}}$ & $6.23^{\mathrm{c}}$ \\
$\mathrm{T}_{3} \mathrm{HE}$ & $8.85^{\mathrm{a}}$ & $7.61^{\mathrm{a}}$ & $7.85^{\mathrm{b}}$ & $8.88^{\mathrm{a}}$ & $7.83^{\mathrm{a}}$ & $7.49^{\mathrm{b}}$ \\
$\mathrm{T}_{4 \mathrm{HE}}$ & $8.91^{\mathrm{a}}$ & $7.75^{\mathrm{a}}$ & $9.64^{\mathrm{a}}$ & $8.95^{\mathrm{a}}$ & $7.98^{\mathrm{a}}$ & $9.68^{\mathrm{a}}$ \\
${ }^{1} \mathrm{LSD}$ & 3.8879 & 2.0971 & 0.6773 & 1.2360 & 1.2027 & 1.2205 \\
${ }^{2} \mathrm{~F}-\mathrm{value}$ & $12.78^{*}$ & $116.95^{*}$ & $319.12^{*}$ & $118.48^{*}$ & $94.48^{*}$ & $66.72^{*}$ \\
\hline
\end{tabular}

Means followed by different letters within one column differ significantly at $P<5 \%$

* Significant at $P<1 \%$ 
The maximum (98\%) radical length was observed for T. aestivum, $P$. minor, E. helioscopia and A. fatua. The final data concluded that minimum radical length was noted for C. album (60\%) and R. dentatus (50\%) on filter paper and soil, respectively (Fig. $2 b$ ).

\section{Plumule length}

$P$. roxburghii hexane extract significantly retarded the plumule length of $A$. fatua (38\%) followed by $R$. dentatus (34\%) as compared to control on filter paper (Table 11), whereas, no momentous effect on plumule length of T. aestivum, P. minor, E. helioscopia and $C$. album. Likewise, the plumule length of $R$. dentatus (49\%) and A. fatua (45\%) was suppressed significantly as compared to control in extract applied into soil (Table 12).

Table 11. Allelopathic effect of $P$. roxburghii hexane extract $(H E)$ on plumule length $(\mathrm{cm})$ of test species on filter paper

\begin{tabular}{c|c|c|c|c|c|c}
\hline \multirow{2}{*}{ Treatments } & \multicolumn{6}{|c}{ Test species } \\
\cline { 2 - 6 } & T. aestivum & A. fatua & R. dentatus & $\boldsymbol{P}$. minor & E. helioscopia & C. album \\
\hline $\mathrm{T}_{1 \mathrm{HE}}$ & $8.08^{\mathrm{a}}$ & $5.63^{\mathrm{d}}$ & $5.41^{\mathrm{c}}$ & $7.14^{\mathrm{a}}$ & $7.06^{\mathrm{a}}$ & $8.12^{\mathrm{a}}$ \\
$\mathrm{T}_{2 \mathrm{HE}}$ & $8.11^{\mathrm{a}}$ & $6.78^{\mathrm{c}}$ & $6.01^{\mathrm{c}}$ & $7.17^{\mathrm{a}}$ & $7.08^{\mathrm{a}}$ & $8.29^{\mathrm{a}}$ \\
$\mathrm{T}_{3 \mathrm{HE}}$ & $8.15^{\mathrm{a}}$ & $7.89^{\mathrm{b}}$ & $7.11^{\mathrm{b}}$ & $7.19^{\mathrm{a}}$ & $7.10^{\mathrm{a}}$ & $8.31^{\mathrm{a}}$ \\
$\mathrm{T}_{4} \mathrm{HE}$ & $8.18^{\mathrm{a}}$ & $9.04^{\mathrm{a}}$ & $8.15^{\mathrm{a}}$ & $7.22^{\mathrm{a}}$ & $7.11^{\mathrm{a}}$ & $8.33^{\mathrm{a}}$ \\
${ }^{1} \mathrm{LSD}$ & 2.3332 & 1.4291 & 0.8679 & 1.2428 & 0.7319 & 0.8297 \\
${ }^{2} \mathrm{~F}$-value & $27.95^{*}$ & $23.83^{*}$ & $81.03^{*}$ & $29.85^{*}$ & $219.87^{*}$ & $222.41^{*}$ \\
\hline
\end{tabular}

Means followed by different letters within one column differ significantly at $P<5 \%$

*Significant at $P<1 \%$

Table 12. Allelopathic effect of $P$. roxburghii hexane extract $(H E)$ on plumule length $(\mathrm{cm})$ of test species on soil

\begin{tabular}{c|c|c|c|c|c|c}
\hline \multirow{2}{*}{ Treatments } & \multicolumn{6}{|c}{ Test species } \\
\cline { 2 - 6 } & T. aestivum & A. fatua & R. dentatus & P. minor & E. helioscopia & C. album \\
\hline $\mathrm{T}_{1 \mathrm{HE}}$ & $8.49^{\mathrm{a}}$ & $5.18^{\mathrm{c}}$ & $4.42^{\mathrm{d}}$ & $7.54^{\mathrm{a}}$ & $7.43^{\mathrm{a}}$ & $8.64^{\mathrm{a}}$ \\
$\mathrm{T}_{2} \mathrm{HE}$ & $8.51^{\mathrm{a}}$ & $6.72^{\mathrm{b}}$ & $5.53^{\mathrm{c}}$ & $7.69^{\mathrm{a}}$ & $7.52^{\mathrm{a}}$ & $8.73^{\mathrm{a}}$ \\
$\mathrm{T}_{3 \mathrm{HE}}$ & $8.62^{\mathrm{a}}$ & $7.41^{\mathrm{b}}$ & $6.67^{\mathrm{b}}$ & $7.79^{\mathrm{a}}$ & $7.62^{\mathrm{a}}$ & $8.88^{\mathrm{a}}$ \\
$\mathrm{T}_{4} \mathrm{HE}$ & $8.78^{\mathrm{a}}$ & $9.39^{\mathrm{a}}$ & $8.75^{\mathrm{a}}$ & $7.82^{\mathrm{a}}$ & $7.71^{\mathrm{a}}$ & $8.93^{\mathrm{a}}$ \\
${ }^{1} \mathrm{LSD}$ & 2.3501 & 0.8878 & 0.7630 & 1.6434 & 1.3019 & 0.5319 \\
${ }^{2} \mathrm{~F}-\mathrm{value}$ & $9.65^{* *}$ & $75.85^{*}$ & $101.54^{*}$ & $73.1^{*}$ & $84.41^{*}$ & $239.87^{*}$ \\
\hline
\end{tabular}

Means followed by different letters within one column differ significantly at $P<5 \%$

*Significant at $P<1 \%$

The maximum plumule length (98\%) was noted for T. aestivum, P. minor, E. helioscopia and $C$. album. The statistical data concluded that minimum plumule length was noted for A. fatua (62\%) and $R$. dentatus (61\%) on filter paper and soil, respectively (Fig. 2c).

\section{Allelopathic potential of $P$. roxburghii methanolic extract}

\section{Germination percentage}

P. roxburghii methanolic extract on filter paper showed significant inhibitory activity on seed germination of T. aestivum (57\%), C. album (49\%), and A. fatua (46\%), respectively as compared to control (Table 13). Likewise, $P$. roxburghii methanolic extract on soil exhibited the highest degree of inhibition germination for T. aestivum 
(62\%), C. album (52\%), and A. fatua (49\%) respectively as compared to control (Table 14). The statistical data also suggested that the there was no significant effect on germination \%age of $P$. minor, R. dentatus and E. helioscopia. The maximum (98\%) germination was observed for $P$. minor, $R$. dentatus and E. helioscopia. The statistical results recommended that minimum germination was noted for $T$. aestivum showing $43 \%$ and $38 \%$ on filter paper and soil, respectively. The statistics also recommended that allelopathic inhibitory effect was concentration dependent for $T$. aestivum, $C$. album and A. fatua (Fig. 3a).

Table 13. Allelopathic effect of $P$. roxburghii methanolic extract $(M E)$ on germination percentage of test species on filter paper

\begin{tabular}{c|c|c|c|c|c|c}
\hline \multirow{2}{*}{ Treatments } & \multicolumn{6}{|c}{ Test species } \\
\cline { 2 - 7 } & T. aestivum & A. fatua & R. dentatus & P. minor & E. helioscopia & C. album \\
\hline $\mathrm{T}_{1 \mathrm{ME}}$ & $40^{\mathrm{d}}$ & $49^{\mathrm{d}}$ & $82^{\mathrm{a}}$ & $84^{\mathrm{a}}$ & $86^{\mathrm{a}}$ & $47^{\mathrm{d}}$ \\
$\mathrm{T}_{2 \mathrm{ME}}$ & $64^{\mathrm{c}}$ & $69^{\mathrm{c}}$ & $84^{\mathrm{a}}$ & $86^{\mathrm{a}}$ & $88^{\mathrm{a}}$ & $62^{\mathrm{c}}$ \\
$\mathrm{T}_{3 \mathrm{ME}}$ & $83^{\mathrm{b}}$ & $80^{\mathrm{b}}$ & $85^{\mathrm{a}}$ & $87^{\mathrm{a}}$ & $89^{\mathrm{a}}$ & $77^{\mathrm{b}}$ \\
$\mathrm{T}_{4 \mathrm{ME}}$ & $93^{\mathrm{a}}$ & $91^{\mathrm{a}}$ & $86^{\mathrm{a}}$ & $88^{\mathrm{a}}$ & $90^{\mathrm{a}}$ & $93^{\mathrm{a}}$ \\
${ }^{1} \mathrm{LSD}$ & 18.606 & 17.890 & 17.535 & 18.50 & 19.67 & 21.68 \\
${ }^{2} \mathrm{~F}-\mathrm{value}$ & $10.29^{* *}$ & $18.19^{*}$ & $23.85^{*}$ & $33.69^{*}$ & $33.71^{*}$ & $14.19^{*}$ \\
\hline
\end{tabular}

Means followed by different letters within one column differ significantly at $P<5 \%$

*Significant at $P<1 \%$

Table 14. Allelopathic effect of $P$. roxburghii methanolic extract (ME) on germination percentage of test species on soil

\begin{tabular}{c|c|c|c|c|c|c}
\hline \multirow{2}{*}{ Treatments } & \multicolumn{6}{|c}{ Test species } \\
\cline { 2 - 7 } & T. aestivum & A. fatua & R. dentatus & P. minor & E. helioscopia & C. album \\
\hline $\mathrm{T}_{1 \mathrm{ME}}$ & $36^{\mathrm{d}}$ & $47^{\mathrm{d}}$ & $83^{\mathrm{a}}$ & $85^{\mathrm{a}}$ & $87^{\mathrm{a}}$ & $45^{\mathrm{d}}$ \\
$\mathrm{T}_{2 \mathrm{ME}}$ & $55^{\mathrm{c}}$ & $62^{\mathrm{c}}$ & $85^{\mathrm{a}}$ & $87^{\mathrm{a}}$ & $89^{\mathrm{a}}$ & $59^{\mathrm{c}}$ \\
$\mathrm{T}_{3 \mathrm{ME}}$ & $69^{\mathrm{b}}$ & $77^{\mathrm{b}}$ & $86^{\mathrm{a}}$ & $88^{\mathrm{a}}$ & $90^{\mathrm{a}}$ & $70^{\mathrm{b}}$ \\
$\mathrm{T}_{4 \mathrm{ME}}$ & $94^{\mathrm{a}}$ & $92^{\mathrm{a}}$ & $87^{\mathrm{a}}$ & $89^{\mathrm{a}}$ & $91^{\mathrm{a}}$ & $94^{\mathrm{a}}$ \\
${ }^{1} \mathrm{LSD}$ & 13.722 & 18.26 & 18.774 & 13.74 & 14.529 & 17.77 \\
${ }^{2} \mathrm{~F}-\mathrm{value}$ & $25.64^{*}$ & $22.09^{*}$ & $15.39^{*}$ & $16.90^{*}$ & $20.73^{*}$ & $32.81^{*}$ \\
\hline
\end{tabular}

Means followed by different letters within one column differ significantly at $P<5 \%$

*Significant at $P<1 \%$

\section{Radical length}

The data revealed that the highest radical length inhibition activity exhibited by $R$. dentatus (47\%) followed by C. album (43\%), measuring $47 \%$ and $43 \%$ in P. roxburghii methanolic extract on filter paper (Table 15). Likewise, methanolic extract on soil caused significant radical length reduction of $R$. dentatus and C. album measuring 50\% and $48 \%$, respectively as compared to control, while $T$. aestivum $P$. minor, $E$. helioscopia and A. fatua remained unaffected (Table 16). The maximum radical length was noted for T. aestivum, P. minor, E. helioscopia and A. fatua (98\%). The results also 
illustrated that minimum radical length was noted for $R$. dentatus i.e. 53\% and $50 \%$ on filter paper and soil, respectively. The results revealed that allelopathic inhibitory effect was concentration dependent for R. dentatus and C. album (Fig. 3b).

Table 15. Allelopathic effect of $P$. roxburghii methanolic extract $(M E)$ on radical length $(\mathrm{cm})$ of test species on filter paper

\begin{tabular}{c|c|c|c|c|c|c}
\hline \multirow{2}{*}{ Treatments } & \multicolumn{6}{|c}{ Test species } \\
\cline { 2 - 7 } & T. aestivum & A. fatua & R. dentatus & P. minor & E. helioscopia & C. album \\
\hline $\mathrm{T}_{1 \mathrm{ME}}$ & $8.31^{\mathrm{a}}$ & $7.31^{\mathrm{a}}$ & $4.97^{\mathrm{d}}$ & $8.45^{\mathrm{a}}$ & $7.46^{\mathrm{a}}$ & $5.39^{\mathrm{d}}$ \\
$\mathrm{T}_{2 \mathrm{ME}}$ & $8.45^{\mathrm{a}}$ & $7.42^{\mathrm{a}}$ & $7.13^{\mathrm{c}}$ & $8.59^{\mathrm{a}}$ & $7.55^{\mathrm{a}}$ & $6.92^{\mathrm{c}}$ \\
$\mathrm{T}_{3 \mathrm{ME}}$ & $8.63^{\mathrm{a}}$ & $7.51^{\mathrm{a}}$ & $8.81^{\mathrm{b}}$ & $8.67^{\mathrm{a}}$ & $7.69^{\mathrm{a}}$ & $8.17^{\mathrm{b}}$ \\
$\mathrm{T}_{4 \mathrm{ME}}$ & $8.71^{\mathrm{a}}$ & $7.55^{\mathrm{a}}$ & $9.44^{\mathrm{a}}$ & $8.75^{\mathrm{a}}$ & $7.78^{\mathrm{a}}$ & $9.48^{\mathrm{a}}$ \\
${ }^{1} \mathrm{LSD}$ & 3.7087 & 1.2858 & 0.7098 & 0.7741 & 0.9677 & 0.998 \\
${ }^{2} \mathrm{~F}-\mathrm{value}$ & $45.38^{*}$ & $99.75^{*}$ & $216.14^{*}$ & $151.39^{*}$ & $124.19^{*}$ & $64.33^{*}$ \\
\hline
\end{tabular}

Means followed by different letters within one column differ significantly at $P<5 \%$

*Significant at $P<1 \%$

Table 16. Allelopathic effect of $P$. roxburghii methanolic extract $(M E)$ on radical length $(\mathrm{cm})$ of test species on soil

\begin{tabular}{c|c|c|c|c|c|c}
\hline \multirow{2}{*}{ Treatments } & \multicolumn{6}{|c}{ Test species } \\
\cline { 2 - 7 } & T. aestivum & A. fatua & R. dentatus & P. minor & E. helioscopia & C. album \\
\hline $\mathrm{T}_{1 \mathrm{ME}}$ & $8.66^{\mathrm{a}}$ & $7.42^{\mathrm{a}}$ & $4.79^{\mathrm{d}}$ & $8.63^{\mathrm{a}}$ & $7.65^{\mathrm{a}}$ & $5.04^{\mathrm{d}}$ \\
$\mathrm{T}_{2 \mathrm{ME}}$ & $8.73^{\mathrm{a}}$ & $7.52^{\mathrm{a}}$ & $6.11^{\mathrm{c}}$ & $8.78^{\mathrm{a}}$ & $7.74^{\mathrm{a}}$ & $6.23^{\mathrm{c}}$ \\
$\mathrm{T}_{3 \mathrm{ME}}$ & $8.85^{\mathrm{a}}$ & $7.61^{\mathrm{a}}$ & $7.85^{\mathrm{b}}$ & $8.88^{\mathrm{a}}$ & $7.83^{\mathrm{a}}$ & $7.49^{\mathrm{b}}$ \\
$\mathrm{T}_{4 \mathrm{ME}}$ & $8.91^{\mathrm{a}}$ & $7.75^{\mathrm{a}}$ & $9.64^{\mathrm{a}}$ & $8.95^{\mathrm{a}}$ & $7.98^{\mathrm{a}}$ & $9.68^{\mathrm{a}}$ \\
${ }^{1} \mathrm{LSD}$ & 3.8879 & 2.0971 & 0.6773 & 1.2360 & 1.2027 & 1.2205 \\
${ }^{2} \mathrm{~F}-\mathrm{v}$-value & $12.78^{*}$ & $116.95^{*}$ & $319.12^{*}$ & $118.48^{*}$ & $94.48^{*}$ & $66.72^{*}$ \\
\hline
\end{tabular}

Means followed by different letters within one column differ significantly at $P<5 \%$

*Significant at $P<1 \%$

\section{Plumule length}

$P$. roxburghii methanolic extract on filter paper significantly inhibited plumule length of $R$. dentatus (46\%) and A. fatua (42\%) as compared to the control (Table 17). Likewise, the highest degree of inhibition in plumule length was measured for $R$. dentatus (49\%) and A. fatua (45\%) in methanolic extract applied into soil (Table 18). The data further suggested that there was no significant effect on germination of $T$. aestivum, $P$. minor, E. helioscopia and C. album. The statistical results recommended that highest plumule length $(98 \%)$ was exhibited by $T$. aestivum, $P$. minor, $E$. helioscopia and $C$. album. The results further indicated that minimum plumule length noticed for R. dentatus i.e. $68 \%$ and $61 \%$ on filter paper and soil, respectively (Fig. 3c). 
Table 17. Allelopathic effect of $P$. roxburghii methanolic extract (ME) on plumule length (cm) of test species on filter paper

\begin{tabular}{c|c|c|c|c|c|c}
\hline \multirow{2}{*}{ Treatments } & \multicolumn{6}{|c}{ Test species } \\
\cline { 2 - 7 } & T. aestivum & A. fatua & R. dentatus & P. minor & E. helioscopia & C. album \\
\hline $\mathrm{T}_{1 \mathrm{ME}}$ & $8.18^{\mathrm{a}}$ & $5.31^{\mathrm{d}}$ & $4.53^{\mathrm{d}}$ & $7.39^{\mathrm{a}}$ & $7.14^{\mathrm{a}}$ & $8.34^{\mathrm{a}}$ \\
$\mathrm{T}_{2 \mathrm{ME}}$ & $8.29^{\mathrm{a}}$ & $7.18^{\mathrm{c}}$ & $6.12^{\mathrm{c}}$ & $7.43^{\mathrm{a}}$ & $7.29^{\mathrm{a}}$ & $8.43^{\mathrm{a}}$ \\
$\mathrm{T}_{3 \mathrm{ME}}$ & $8.35^{\mathrm{a}}$ & $8.51^{\mathrm{b}}$ & $7.23^{\mathrm{b}}$ & $7.51^{\mathrm{a}}$ & $7.35^{\mathrm{a}}$ & $8.55^{\mathrm{a}}$ \\
$\mathrm{T}_{4 \mathrm{ME}}$ & $8.48^{\mathrm{a}}$ & $9.14^{\mathrm{a}}$ & $8.45^{\mathrm{a}}$ & $7.52^{\mathrm{a}}$ & $7.41^{\mathrm{a}}$ & $8.63^{\mathrm{a}}$ \\
${ }^{1} \mathrm{LSD}$ & 2.1332 & 1.5092 & 0.6679 & 1.5428 & 0.7497 & 0.6297 \\
${ }^{2} \mathrm{~F}-\mathrm{value}$ & $28.55^{*}$ & $24.81^{*}$ & $84.03^{*}$ & $27.95^{*}$ & $220.41^{*}$ & $221.91^{*}$ \\
\hline
\end{tabular}

Means followed by different letters within one column differ significantly at $P<5 \%$

*Significant at $P<1 \%$

Table 18. Allelopathic effect of $P$. roxburghii methanolic extract (ME) on plumule length (cm) of test species on soil

\begin{tabular}{c|c|c|c|c|c|c}
\hline \multirow{2}{*}{ Treatments } & \multicolumn{6}{|c}{ Test species } \\
\cline { 2 - 7 } & T. aestivum & A. fatua & R. dentatus & P. minor & E. helioscopia & C. album \\
\hline $\mathrm{T}_{1 \mathrm{ME}}$ & $8.49^{\mathrm{a}}$ & $5.18^{\mathrm{c}}$ & $4.42^{\mathrm{d}}$ & $7.54^{\mathrm{a}}$ & $7.43^{\mathrm{a}}$ & $8.64^{\mathrm{a}}$ \\
$\mathrm{T}_{2 \mathrm{ME}}$ & $8.51^{\mathrm{a}}$ & $6.72^{\mathrm{b}}$ & $5.53^{\mathrm{c}}$ & $7.69^{\mathrm{a}}$ & $7.52^{\mathrm{a}}$ & $8.73^{\mathrm{a}}$ \\
$\mathrm{T}_{3 \mathrm{ME}}$ & $8.62^{\mathrm{a}}$ & $7.41^{\mathrm{b}}$ & $6.67^{\mathrm{b}}$ & $7.79^{\mathrm{a}}$ & $7.62^{\mathrm{a}}$ & $8.88^{\mathrm{a}}$ \\
$\mathrm{T}_{4 \mathrm{ME}}$ & $8.78^{\mathrm{a}}$ & $9.39^{\mathrm{a}}$ & $8.75^{\mathrm{a}}$ & $7.82^{\mathrm{a}}$ & $7.71^{\mathrm{a}}$ & $8.93^{\mathrm{a}}$ \\
${ }^{1} \mathrm{LSD}$ & 2.3501 & 0.8878 & 0.7630 & 1.6434 & 1.3019 & 0.5319 \\
${ }^{2} \mathrm{~F}-\mathrm{value}$ & $9.65^{* *}$ & $75.85^{*}$ & $101.54^{*}$ & $73.1^{*}$ & $84.41^{*}$ & $239.87^{*}$ \\
\hline
\end{tabular}

Means followed by different letters within one column differ significantly at $P<5 \%$

*Significant at $P<1 \%$

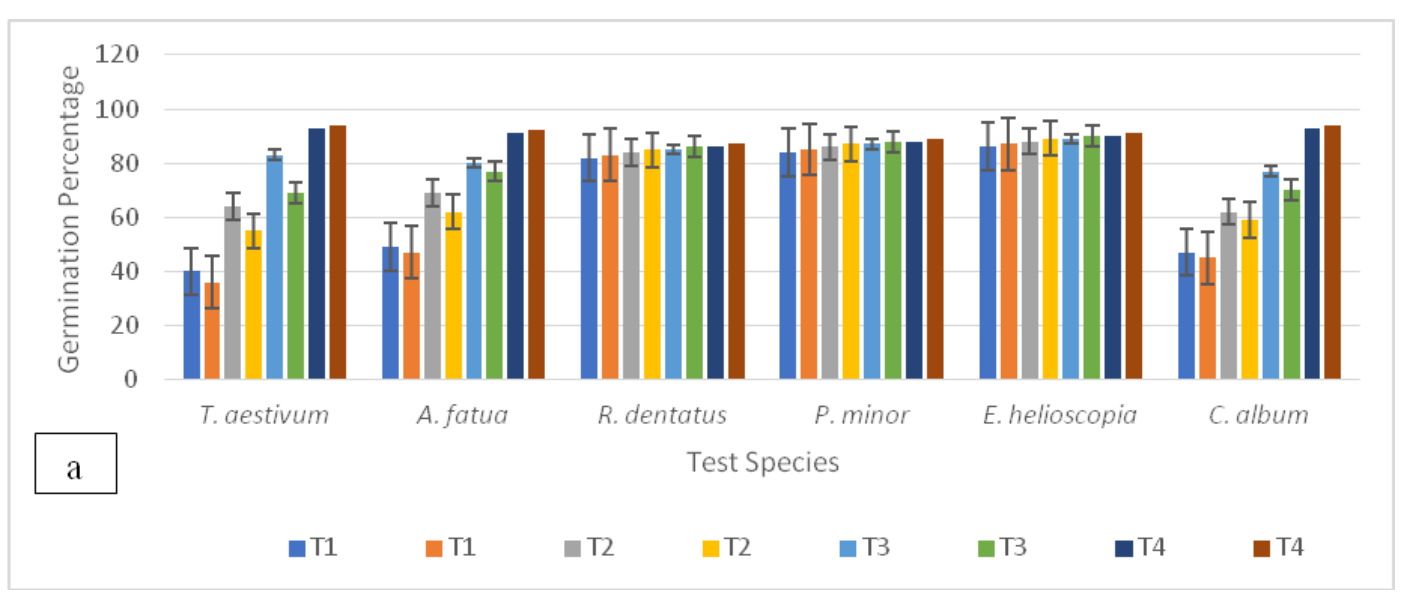




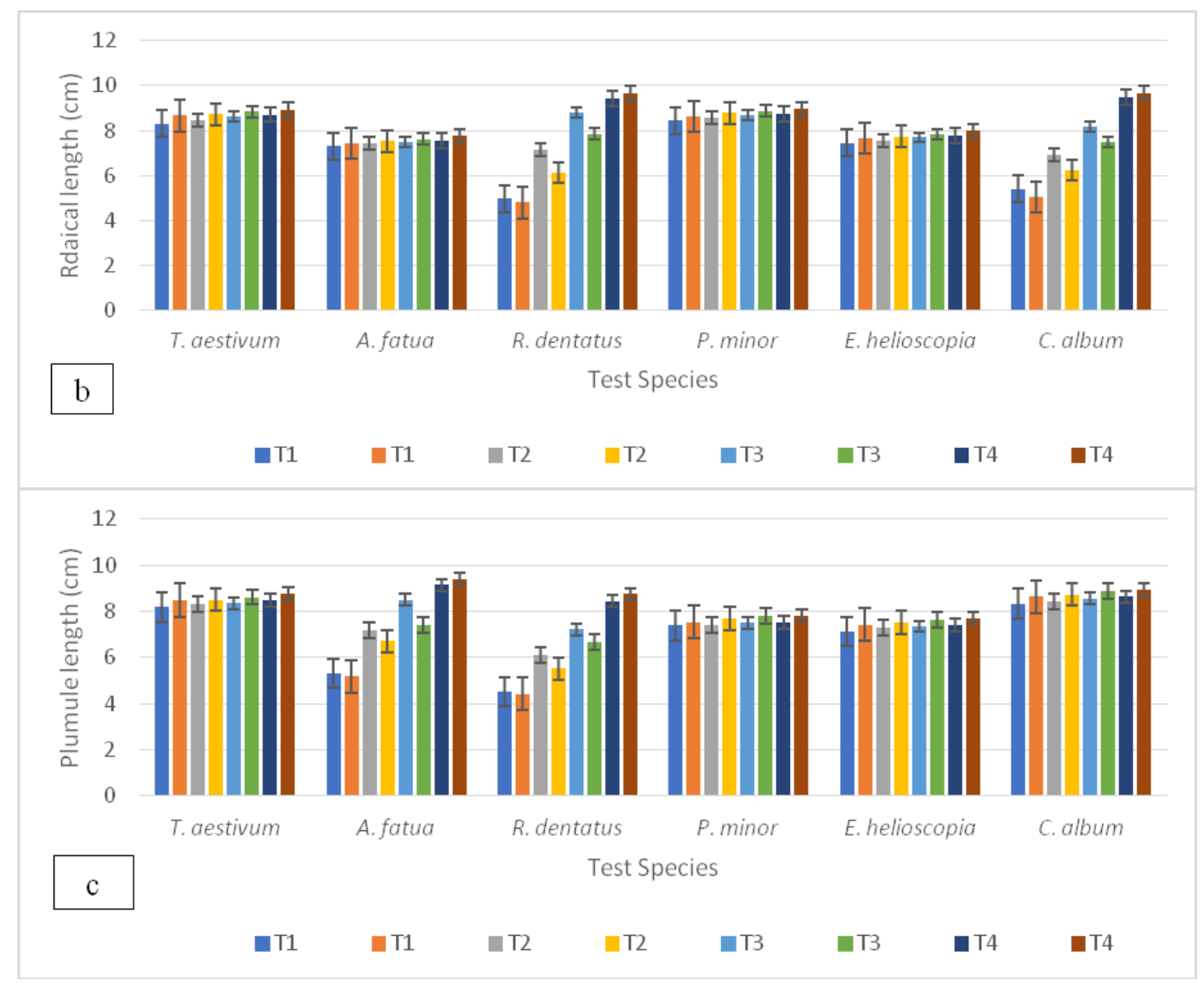

Figure 3. Allelopathic potential of $P$. roxburghii methanolic extract against test species on filter paper $(F P)$ and soil $(S)$ on: (a) germination percentage $(b)$ radical length $(c)$ plumule length $\left(T_{1}\right.$ $=100 \%, T_{2}=75 \%, T_{3}=50 \%$ and $T_{4}=$ control $)$

\section{Allelopathic potential of $P$. roxburghii in sandwich method}

\section{Germination percentage}

The data revealed that $T$. aestivum, $C$. album and A. fatua showing $52 \%, 47 \%$ and $44 \%$ germination inhibition respectively as compared to control in $P$. roxburghii needles powder on filter paper, whereas, no significant effect on germination of $R$. dentatus, $P$. minor and E. helioscopia showing resistance to dry powder. The results also declared that maximum (97\%) germination was noted for $R$. dentatus, P. minor and E. helioscopia. In the present study, it was demonstrated that minimum germination was noted for T. aestivum i.e. $48 \%$ and $45 \%$ on filter paper and soil, respectively. The experimental results of the current study indicated on agar the highest germination reduction was noted for T. aestivum (51\%), followed by C. album (36\%) and A. fatua $(35 \%)$ at $10 \mathrm{mg}$ conc. Similarly, the highest germination reduction was noted for $T$. aestivum (56\%), followed by C. album (48\%) and A. fatua (43\%) at $50 \mathrm{mg}$ conc (Table 19). The statistical data concluded that minimum germination was noted for $T$. aestivum measuring $49 \%$ and $44 \%$ at $10 \mathrm{mg}$ and at $50 \mathrm{mg}$ conc., respectively. The statistics also recommended that with the increase of concentration, the inhibitory effect was progressively increased for T. aestivum, C. album and A. fatua. The statistical 
results recommended that the germination of $P$. minor, E. helioscopia and $R$. dentatus were completely resistant to dry powder (Fig. 4a).

Table 19. Allelopathic effect of $P$. roxburghii leaf powder on germination percentage of test species

\begin{tabular}{|c|c|c|c|c|c|c|c|}
\hline \multicolumn{2}{|c|}{ Treatments } & \multicolumn{6}{|c|}{ Test species } \\
\hline Media & & T. aestivum & A. fatua & R. dentatus & P. minor & E. helioscopia & C. album \\
\hline \multirow{2}{*}{$\begin{array}{l}\text { Filter } \\
\text { paper }\end{array}$} & $10 \mathrm{mg}$ & $43^{\mathrm{b}}$ & $44^{\mathrm{b}}$ & $86^{\mathrm{a}}$ & $74^{\mathrm{a}}$ & $83^{\mathrm{a}}$ & $48^{\mathrm{b}}$ \\
\hline & Control & $90^{\mathrm{a}}$ & $78^{a}$ & $88^{\mathrm{a}}$ & $79^{\mathrm{a}}$ & $84^{\mathrm{a}}$ & $91^{\mathrm{a}}$ \\
\hline \multirow{2}{*}{ Soil } & $50 \mathrm{mg}$ & $41^{\mathrm{b}}$ & $43^{\mathrm{b}}$ & $87^{\mathrm{a}}$ & $80^{\mathrm{a}}$ & $85^{\mathrm{a}}$ & $45^{\mathrm{b}}$ \\
\hline & Control & $91^{\mathrm{a}}$ & $80^{\mathrm{a}}$ & $90^{\mathrm{a}}$ & $81^{\mathrm{a}}$ & $86^{\mathrm{a}}$ & $93^{\mathrm{a}}$ \\
\hline \multirow{3}{*}{ Agar } & $10 \mathrm{mg}$ & $46^{\mathrm{b}}$ & $54^{\mathrm{b}}$ & $93^{\mathrm{a}}$ & $82^{\mathrm{a}}$ & $88^{\mathrm{a}}$ & $60^{\mathrm{b}}$ \\
\hline & $50 \mathrm{mg}$ & $41^{\mathrm{b}}$ & $47^{\mathrm{b}}$ & $91^{\mathrm{a}}$ & $80^{\mathrm{a}}$ & $86^{\mathrm{a}}$ & $49^{c}$ \\
\hline & Control & $93^{a}$ & $83^{\mathrm{a}}$ & $93^{\mathrm{a}}$ & $83^{\mathrm{a}}$ & $89^{\mathrm{a}}$ & $95^{\mathrm{a}}$ \\
\hline \multicolumn{2}{|c|}{${ }^{21} \mathrm{LSD}$} & 13.36 & 18.44 & 19.53 & 18.957 & 23.14 & 15.64 \\
\hline \multicolumn{2}{|c|}{${ }^{2} \mathrm{~F}$-value } & $16.69 *$ & $14.95 *$ & $31.64 *$ & $32.00 *$ & $19.62 *$ & $16.2 *$ \\
\hline
\end{tabular}

Means followed by different letters within one column differ significantly at $P<5 \%$

*Significant at $P<1 \%$

\section{Radical length}

The data revealed that $C$. album and $R$. dentatus showing $35 \%$ and $32 \%$ radical length inhibition respectively as compared to control in $P$. roxburghii needles powder on filter paper, whereas, no significant effect on radical length of T. aestivum, A. fatua, $P$. minor and E. helioscopia showing resistance to dry powder. It is also clear from the result that $C$. album and $R$. dentatus showed $45 \%$ and $42 \%$ radical length inhibition respectively as compared to control in powder applied into soil. The results also declared that maximum (96\%) radical length was noted for T. aestivum, A. fatua, P. minor and E. helioscopia. In the present study, it was demonstrated that minimum radical length was noted for $C$. album i.e. $65 \%$ and $55 \%$ on filter paper and soil, respectively (Table 20). The experimental results of the current study indicated on agar the highest radical length reduction was noted for $R$. dentatus $(35 \%)$, followed by $C$. album (34\%) at $10 \mathrm{mg}$ conc. Similarly, the highest radical length reduction was noted for $R$. dentatus ( $41 \%$ ), followed by $C$. album (36\%) at $50 \mathrm{mg}$ conc. The statistical data concluded that minimum radical length was noted for $R$. dentatus i.e. $65 \%$ and $69 \%$ at $10 \mathrm{mg}$ and at $50 \mathrm{mg}$ conc., respectively (Fig. $4 b$ ).

\section{Plumule length}

The data revealed that $A$. fatua (39\%) and $R$. dentatus (37\%) showing plumule length inhibition respectively as compared to control in $P$. roxburghii needles powder on filter paper, whereas, no significant effect on plumule length of T. aestivum, C. album, E. helioscopia and $P$. minor showing resistance to dry powder. It is also clear from the result that $R$. dentatus (49\%) and A. fatua (46\%) showed and plumule length inhibition respectively as compared to control in powder applied into soil. The results also declared that maximum (95\%) plumule length was noted for T. aestivum, C. album, E. 
helioscopia and $P$. minor. In the present study, it was demonstrated that minimum plumule length was noted for A. fatua $(61 \%)$ and $R$. dentatus $(51 \%)$ on filter paper and soil, respectively (Table 21). The results of the current study indicated on agar the highest plumule length reduction was noted for $R$. dentatus (42\%), followed by A. fatua (40\%) at $10 \mathrm{mg}$ conc. Similarly, the highest plumule length reduction was noted for $R$. dentatus (49\%), followed by A. fatua (44\%) at $50 \mathrm{mg}$ conc. The statistical data concluded that minimum plumule length was noted for $R$. dentatus measuring $68 \%$ and $61 \%$ at $10 \mathrm{mg}$ and at $50 \mathrm{mg}$ conc., respectively (Fig. $4 \mathrm{c}$ ).

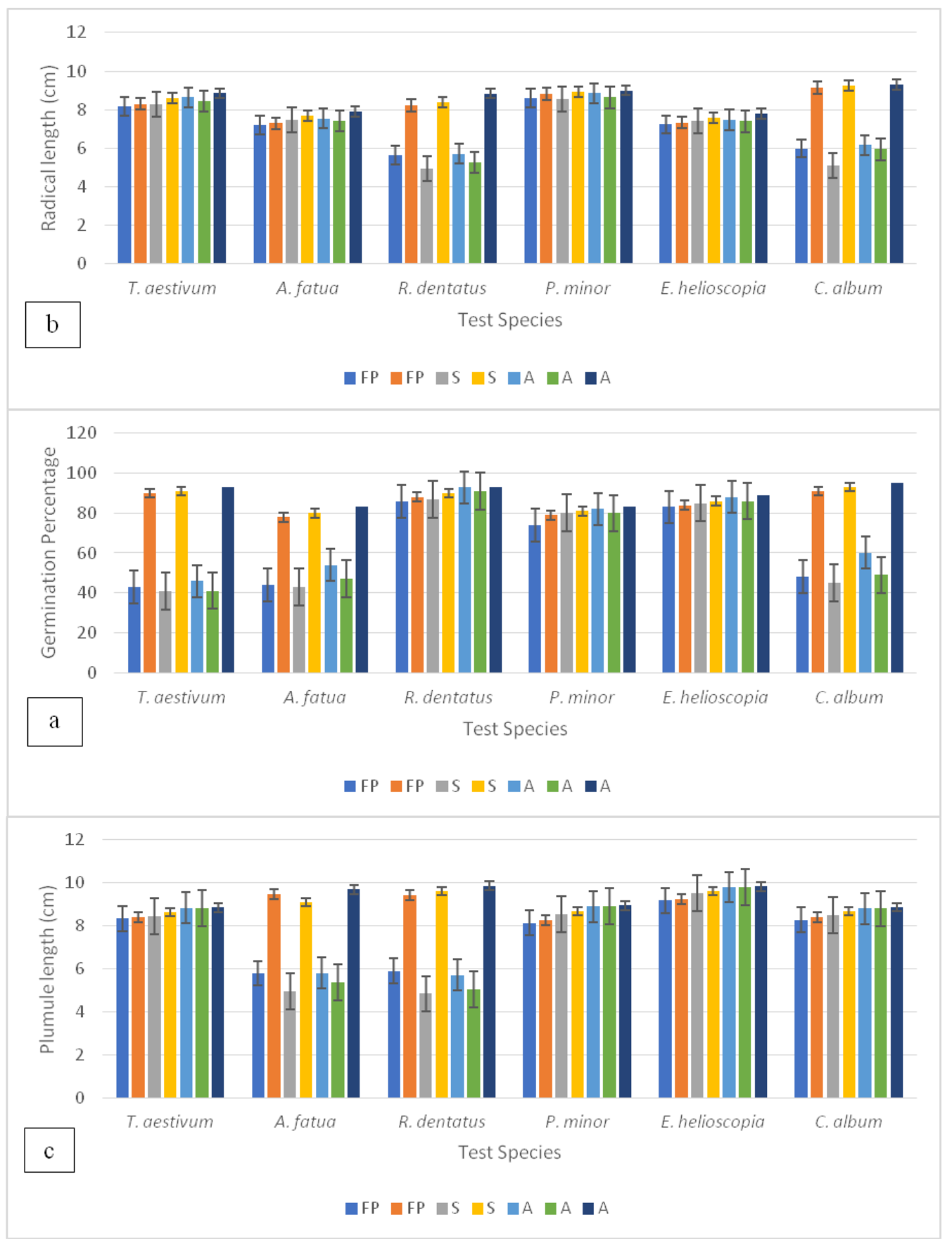

Figure 4. Allelopathic potential of $P$. roxburghii needles powder against test species on filter paper $(F P)$ and soil $(S)$ on: (a) germination percentage (b) radical length (c) plumule length $\left(T_{1}\right.$ $=100 \%, T_{2}=75 \%, T_{3}=50 \%$ and $T_{4}=$ control $)$ 
Table 20. Allelopathic effect of $P$. roxburghii leaf powder on radical length (cm) of test species

\begin{tabular}{c|c|c|c|c|c|c|c}
\hline \multirow{2}{*}{ Treatments } & \multicolumn{6}{c}{ Test species } \\
\cline { 3 - 8 } & \multirow{2}{*}{ T. aestivum } & A. fatua & R. dentatus & P. minor & E. helioscopia & C. album \\
\hline Filter & $10 \mathrm{mg}$ & $8.16^{\mathrm{a}}$ & $7.21^{\mathrm{a}}$ & $5.63^{\mathrm{b}}$ & $8.60^{\mathrm{a}}$ & $7.23^{\mathrm{a}}$ & $5.98^{\mathrm{b}}$ \\
paper & Control & $8.29^{\mathrm{a}}$ & $7.29^{\mathrm{a}}$ & $8.23^{\mathrm{a}}$ & $8.82^{\mathrm{a}}$ & $7.33^{\mathrm{a}}$ & $9.15^{\mathrm{a}}$ \\
\hline \multirow{2}{*}{ Soil } & $50 \mathrm{mg}$ & $8.27^{\mathrm{a}}$ & $7.46^{\mathrm{a}}$ & $4.91^{\mathrm{b}}$ & $8.54^{\mathrm{a}}$ & $7.41^{\mathrm{a}}$ & $5.09^{\mathrm{b}}$ \\
& Control & $8.61^{\mathrm{a}}$ & $7.68^{\mathrm{a}}$ & $8.40^{\mathrm{a}}$ & $8.93^{\mathrm{a}}$ & $7.56^{\mathrm{a}}$ & $9.23^{\mathrm{a}}$ \\
\hline \multirow{4}{*}{ Agar } & $10 \mathrm{mg}$ & $8.64^{\mathrm{a}}$ & $7.54^{\mathrm{a}}$ & $5.71^{\mathrm{b}}$ & $8.85^{\mathrm{a}}$ & $7.46^{\mathrm{a}}$ & $6.15^{\mathrm{b}}$ \\
& $50 \mathrm{mg}$ & $8.42^{\mathrm{a}}$ & $7.41^{\mathrm{a}}$ & $5.25^{\mathrm{b}}$ & $8.63^{\mathrm{a}}$ & $7.39^{\mathrm{a}}$ & $5.93^{\mathrm{b}}$ \\
& Control & $8.85^{\mathrm{a}}$ & $7.89^{\mathrm{a}}$ & $8.83^{\mathrm{a}}$ & $8.99^{\mathrm{a}}$ & $7.78^{\mathrm{a}}$ & $9.31^{\mathrm{a}}$ \\
\hline \multicolumn{2}{c}{${ }^{21}$ LSD } & 3.1879 & 1.9971 & 0.6373 & 1.6360 & 1.7027 & 1.0523 \\
\hline \multicolumn{2}{c}{${ }^{2}$ F-value } & $14.78^{*}$ & $132.95^{*}$ & $301.12^{*}$ & $126.48^{*}$ & $95.48^{*}$ & $48.12^{*}$ \\
\hline
\end{tabular}

Means followed by different letters within one column differ significantly at $P<5 \%$

*Significant at $P<1 \%$

Table 21. Allelopathic effect of $P$. roxburghii leaf powder on plumule length (cm) of test species

\begin{tabular}{|c|c|c|c|c|c|c|c|}
\hline \multirow{2}{*}{\multicolumn{2}{|c|}{ Treatments }} & \multicolumn{6}{|c|}{ Test species } \\
\hline & & \multirow{2}{*}{ T. aestivum } & \multirow{2}{*}{ A. fatua } & \multirow{2}{*}{ R. dentatus } & \multirow{2}{*}{ P. minor } & \multirow{2}{*}{ E. helioscopia } & \multirow{2}{*}{ C. album } \\
\hline Media & & & & & & & \\
\hline \multirow{2}{*}{$\begin{array}{l}\text { Filter } \\
\text { paper }\end{array}$} & $10 \mathrm{mg}$ & $8.35^{\mathrm{a}}$ & $5.80^{\mathrm{b}}$ & $5.91^{\mathrm{b}}$ & $8.15^{\mathrm{a}}$ & $9.19^{\mathrm{a}}$ & $8.28^{\mathrm{a}}$ \\
\hline & Control & $8.41^{\mathrm{a}}$ & $9.48^{\mathrm{a}}$ & $9.44^{\mathrm{a}}$ & $8.27^{\mathrm{a}}$ & $9.25^{\mathrm{a}}$ & $8.40^{\mathrm{a}}$ \\
\hline \multirow{2}{*}{ Soil } & $50 \mathrm{mg}$ & $8.46^{\mathrm{a}}$ & $4.95^{b}$ & $4.85^{b}$ & $8.56^{a}$ & $9.52^{\mathrm{a}}$ & $8.49^{a}$ \\
\hline & Control & $8.66^{\mathrm{a}}$ & $9.09^{\mathrm{a}}$ & $9.60^{\mathrm{a}}$ & $8.68^{a}$ & $9.62^{a}$ & $8.68^{a}$ \\
\hline \multirow{3}{*}{ Agar } & $10 \mathrm{mg}$ & $8.84^{\mathrm{a}}$ & $5.82^{b}$ & $5.72^{b}$ & $8.90^{a}$ & $9.80^{\mathrm{a}}$ & $8.82^{a}$ \\
\hline & $50 \mathrm{mg}$ & $8.82^{\mathrm{a}}$ & $5.38^{\mathrm{b}}$ & $5.05^{\mathrm{b}}$ & $8.92^{\mathrm{a}}$ & $9.82^{\mathrm{a}}$ & $8.81^{\mathrm{a}}$ \\
\hline & Control & $8.86^{\mathrm{a}}$ & $9.69^{a}$ & $9.86^{\mathrm{a}}$ & $8.95^{\mathrm{a}}$ & $9.84^{\mathrm{a}}$ & $8.87^{\mathrm{a}}$ \\
\hline \multicolumn{2}{|c|}{${ }^{21} \mathrm{LSD}$} & 4.6846 & 1.5971 & 0.9340 & 0.8018 & 0.3182 & 1.5127 \\
\hline \multicolumn{2}{|c|}{${ }^{2} \mathrm{~F}$-value } & $7.14 * *$ & $43.74 *$ & $55.31 *$ & $55.01 *$ & $14.14^{*}$ & $75.45^{*}$ \\
\hline
\end{tabular}

Means followed by different letters within one column differ significantly at $P<5 \%$

*Significant at $P<1 \%$

\section{Discussion}

Aqueous extracts of Pinus albicaulis, $P$. contorta, $P$ sabiniana and $P$. ponderosa needles inhibited radical length of Hordeum vulgare and Bromus mollis (Heisey and Delwiche, 1983). P. densiflora needles water extract inhibited seed germination and seedling growth in Clematis apiifolia, Ledebouriella seseloides, Melandrium firmum, Bidens pinnata, and Platanus orientalis (Kil and Yim, 1983). Aqueous extracts of Pinus divaricata and $P$. resinosa fresh needles and litter reduced seed germination, root elongation and growth of Poa pratense, Epilobium angustifolium, Agropyron repens and Phleum pratense (Jobidon, 1986). P. koraiensis water extracts from leaves affected the seed germination and seedling elongation of selected weeds of wheat crops (Kil et 
al., 1991). P. sylvestris water soluble root secretions exerted negative biochemical effect on Picea excela (Kolesnichenko and Andryushchenko, 1978) and inhibited germination and early seedling growth of Betula pendula. Betula pubescens and Pinus sylvestris (Hytonen, 1992). Aqueous extracts of $P$. thunbergii needles inhibited seed germination and seedling length of Lactuca sativa, Carpesium abrotanoides and Oenothera odorata (Kil, 1989). The allelopathic potential is due to the presence of $9 \alpha, 13 \beta$-epidioxyabeit8(14)en-18-oic acid reported from the aqueous methanolic extract of red pine needles that inhibited the growth of Lepidium sativum, Lactuca sativa, Medicago sativa, Lolium multiflorum and Digitaria sanguinalis (Kato-Noguchi et al., 2009). Another compound abscisic acid- $\beta$-D-glucopyranosyl ester (ABA-GE) was also isolated and found to have allelopathic activity (Kato-Noguchi et al., 2011). The needle extract from P. nigra inhibited seed germination of rye grass (Terzi, 2013). Similarly, aqueous extract of $P$. roxburghii needles suppressed the growth of mustard and wheat seedlings (Baroniya and Baroniya, 2014), while that of P. brutia needles suppressed the growth of Lolium multiflorum and Poa pratensis seedlings (Aliloo et al., 2012).

The current study was in accordance with Singh et al. (2001), who determined that seedling growth and seed germination of Capsicum annuum, Pisum sativum and Oryza sativa was significantly retarded by Pinus needles and the inhibitory effect was concentration dependent. Likewise, Amaranthus paniculatus and Trifolium pratense seeds treated with leaf extract of $P$. roxburghii and Rhododendron arboreum that resulted momentous consequence on germination of tested species (Madgil and Kapil, 1990). Poisonous compounds produced by $P$. densiflora checked growth and seed germination of adjacent species (Kil and Yim, 1983). The phenolic composites of $P$. rigida exhibited both retardation and development effect on Cassia mimosoides revealing concentration dependent. Fresh, senesced and decaying needles from $P$. halepensis exhibited potent inhibitory potential on Festuca arundinacea, Cyanodon dactylon, Avena sativa and Lemna minor (Nektarios et al., 2005).

Different species of Pinus had exhibited allelopathic potential against other plant species (Kato-Noguchi et al., 2009). P. roxburghii extract possessed significant inhibitory potential on different plants (Melkania, 1984). Allelopathic effects of $P$. halepensis possessed herbicidal activities (Hamrouni et al., 2015; Anwar et al., 2019). A significant herbicidal activity of $P$. halepensis against common weeds of cereal crops (Amri et al., 2013). The inhibitory effect of the Pinus needles, being more pronounced in the fresh, moderate in the senesced, and low in the decaying conditions (Monnier et al., 2011). Allelopathic potential of $P$. halepensis had been broadly calculated from various plant parts, which can be autotoxic and thus prevent the germination of seeds in a forest plants as was observed for inhibition of Stipa tenacissima grasslands (Fernandez et al., 2013; Navarro-Cano et al., 2009). Allelochemicals are reported to be present in stems, roots, leaves and fruit of P. halepensis (Baroniya and Baroniya, 2014), which exhibited allelopathic effects (Hamrouni et al., 2015) and strong herbicidal activity of essential oil was reported against common weeds of cereal crops (Fujii et al., 2004). P. halepensis extracts inhibited germination and growth of Lactuca sativa and Linum strictum. The strong allelophatic potential of $P$. halepensis could be attributed to the presence of numerous phenolic compounds such as benzeneacetic, 4hydroxybenzoic, vanillic, veratric, syringic and p-coumaric acids, and non-phenolic acids such as lactic, succinic, palmitic acids in P. halepensis (Fernandez et al., 2006). Green needles are found to have higher amounts of phenolic compounds and condensed tannins (Refifa et al., 2016). Reduced germination and suppressed growth in different 
plants could be a result of damage in the membrane integrity. It has been observed that seeds supplemented with Pinus needle extract showed enhance electrolyte leakage which reveals higher damage to membranes (Baroniya and Baroniya, 2014).

The black pine showed to inhibit the growth of Phalaris canariensis, Trifolium campestre and Sinapis arvensis seeds (Amri et al., 2013; Anwar et al., 2018c). Different pinene isomers exhibited allelopathic potential against Zea mays seed germination (Areco et al., 2014). The leaf methanolic extract from P. nigra suppressed the seed germination of perennial ryegrass and tall fescue (Robert, 1986; Terzi et al., 2013). Valera-Burgos et al. (2012) noticed inhibitory potential of Pinus pinea needles extract on seedling growth and seed germination 3 Mediterranean shrub species. Allelopatic substance was isolated from the exudates of Japanese red pine trees the substance was identified as phenylacetic acid which inhibited shoot and radical elongation of Cryptantha crassipes I. M. Johnst (Khan et al., 2008; Anwar et al., 2018d). P. densiflora needles contain toxic substances that inhibit seed germination and growth of plants (Fernandez et al., 2013), these inhibitory effects can be attributed to direct molecular alteration (Hamrouni et al., 2015). The main inhibitory substance from methanol extract of the pine needles was identified as 9alpha, 13beta-epidioxyabeit8(14) en-18-oic acid, this substance is responsible for the inhibition of root and shoot growth of Echinochloa crus-galli (Baroniya and Baroniya, 2014).

Powdered $P$. roxburghii needles reduce germination and, root and shoot growth of Achyranthes aspera L. (Khosla et al., 1981; Anwar et al., 2018a). Plants growing at higher altitudes are observed to accumulate phenolic compounds in higher concentrations consequently inhibit plant growth (Baroniya and Baroniya, 2014). Gymnosperm trees showed strong allelopathic effect on the germination, growth, and development of other plant species in the forest community (Silva et al., 2015) due to presence of allelochemicals, mostly phenolic compounds and terpenoids (Rice, 1984). $P$. halepensis had strong inhibitory effect on seedling establishment of various species suggesting allelopathic effects of litter or root exudates (Maestre, et al., 2003). Needle essential oil identified components are $(E)$-caryophyllene, terpinen-4-ol, $\alpha$-humulene, and $\alpha$-terpineol (Satyal et al., 2013; Anwar et al., 2018b), it was reported that $(E)$ caryophyllene inhibited the germination and seedling growth of Brassica campestris and Raphanus sativus (Wang et al., 2009). Application of $\alpha$-pinene inhibited the growth of Cassia occidentalis, Amaranthus viridis, Triticum aestivum, Pisum sativum and Cicer arietinum seedlings due to the oxidative damage in root tissues (Singh et al., 2006). Allelochemicals in chir pine needles suppressed the growth of radical and plumule length of canary grass (Refifa et al., 2016). Blum (1998) observed that $P$. divaricata and $P$. resinosa needles leachates suggestively checked seedling growth and germination of Epilobium angustifolium, Agropyron repens and Phleum pretense. The seedling growth of Lepidium virginicum was significantly checked by $P$. roxburghii needles (Williams and Hoagland, 1982). The mechanism of retardation on the seedling growth produced by phytochemicals checked cell elongation and division (Node et al., 2003). Similarly, $P$. densiflora cones have high biological activity against select plant species (Lee and Monsi, 1963).

\section{Conclusions}

Present results indicated that pine needles extract and dried powder at higher concentrations reduced the seed germination, radical and plumule length of weeds 
associated with the wheat crop. $P$. roxburghii is located in the mountain region of Pakistan and in every season, the fallen needles form a bed on the forest floor. During the rainy season, pine needles get dissolved with water and mixed into the soil and resultantly caused in crop reduction. Further work is needed to appraise the potential inhibitory effects of allelochemicals from the pine needles.

\section{REFERENCES}

[1] Ahmed, S. A., El-Rokiek, K. G., El-Masry, R. R., Messiha, N. K. (2014): The efficiency of allelochemicals in the seed powder of Eruca sativa in controlling weeds in Pisum sativum. - Middle East Journal of Agriculture Research 3: 757-762.

[2] Aliloo, A. A., Shahabivand, S., Farjam, L., Heravi, S. (2012): Allelopathic effects of pine needle extracts on germination and seedling growth of ryegrass and Kentucky bluegrass. - Advances in Environmental Biology 6: 2513-2518.

[3] Amri, I., Hamrouni, L., Hanana, M., Gargouri, S., Fezzani, T., Jamoussi, B. (2013): Chemical composition, physio-chemical properties, antifungal and herbicidal activities of Pinus halepensis Miller essential oils. - Biological Agricultural Horticulture 29: 91-106.

[4] Anwar, T., Khalid, S., Arafat, Y., Sadia, S., Riaz, S. (2013): Allelopathic suppression of Avena fatua and Rumex dentatus in associated crops. - Pakistan Journal of Weed Science Research 19: 31-43.

[5] Anwar, T., Khalid, S., Saeed, M., Mazhar, R., Qureshi, H., Rashid, M. (2016): Allelopathic interference of leaf powder and aqueous extracts of hostile weed: Parthenium hysterophorus (Asteraceae). - Science International 4: 86-93.

[6] Anwar, T., Khalid, S., Mazhar, R., Qureshi, H., Rashid, M. (2017a): Herbicidal potential of selected species to overcome weed infestation in Triticum aestivum, Zea mays and Helianthus annuus. - Pakistan Journal of Weed Science Research 23: 49-63.

[7] Anwar, T., Khalid, S., Panni, M. K., Qureshi, H., Rashid, M. (2017b): Allelopathic effect of Euphorbia helioscopia on Avena fatua, Rumex dentatus, Helianthus annuus, Zea mays and Triticum aestivum. - Pakistan Journal of Weed Science Research 23: 165-177.

[8] Anwar, T., Ilyas, N., Qureshi, R., Munazir, M., Khan, A. M., Ansari, L., Rahim, B. Z., Ansari, K. A., Panni. M. K. (2018a): Allelopathic activity of solvent extracts of Rhazya stricta Decne. against selected weeds of wheat crop. - Applied Ecology and Environmental Research 16(5): 5405-5421.

[9] Anwar, T., Ilyas, N., Qureshi, R., Rahim, B. Z., Maqsood, M., Ansari, K. A., Munazir, M., Panni, M. K. (2018b): Allelopathic potential of Lantana camara against selected weeds of wheat crop. - Applied Ecology and Environmental Research 16(6): 6741-6760.

[10] Anwar, T., Panni, M. K., Khalid, S., Qureshi, H. (2018c): Appraisal of allelopathic potential of curly dock (Rumex dentatus L.) as a natural weed management source. Pakistan Journal of Weed Science Research 24(3): 231-239.

[11] Anwar, T., Panni, M. K., Khalid, S., Qureshi, H. (2018d): Allelopathic management of noxious weeds in Helianthus annuus, Zea mays and Triticum aestivum by selected plants. - Pakistan Journal of Weed Science Research 24(3): 257-265.

[12] Anwar, T., Ilyas, N., Qureshi, R., Malik, M. A. (2019): Allelopathic potential of Carica papaya against selected weeds of wheat crop. - Pakistan Journal of Botany 51(1). DOI: http://dx.doi.org/10.30848/PJB2019-1(37).

[13] Areco, V. A., Figueroa, S., Cosa, M. T., Dambolena, J. S., Zygadlo, J. A., Zunino, M. P. (2014): Effect of pinene isomers on germination and growth of maize. - Biochemical Systematics and Ecology 55: 27-33.

[14] Baroniya, S. S., Baroniya, M. B. (2014): Germination and early seedling growth of mustard and wheat as affected by allelopathic activity of Pinus needle extracts. - Int. Res. J. Environment Sci. 3: 27-34. 
[15] Biljana, M. B., Kragujevac, D. Z. J. (2015): Allelopathic relations of selected cereal and vegetable species during seed germination and seedling growth. - Journal of Science 37: 135-142.

[16] Blum, U. (1998): Effects of microbial utilization of phenolic acids and their phenolic acid breakdown products on allelopathic interactions. - Journal of Chemical Ecology 24: 685708.

[17] Fernandez, C., Vila, B., Mevy, J., Robles, C. (2006): Potential allelopathic effect of Pinus halepensis in the secondary succession: an experimental approach. - Chemoecology 16: 97-105.

[18] Fernandez, C., Santonja, M., Gros, R., Monnier, R. Y., Chomel, M., Baldy, V., Melou, A. B. (2013): Allelochemicals of Pinus halepensis as drivers of biodiversity in Mediterranean open mosaic habitats during the colonization stage of secondary succession. - Journal of Chemical Ecology 39: 298-311.

[19] Fujii, Y., Parvez, S. S., Parvez, M. M., Ohmae, Y., Iida, O. (2003): Screening of 239 medicinal plant species for allelopathic activity using sandwich method. - Weed Biology and Management 3: 233-241.

[20] Fujii, Y., Shibuya, T., Nakatani, K., Itani, T., Hiradate, S., Parvez, M. M. (2004): Assessment method for allelopathic effect from leaf litter leachates. - Weed Biology and Management 4: 19-23.

[21] Hamrouni, L., Hanana, M., Amri, I., Romane, A. E., Gargouri, S., Jamoussi, B. (2015): Allelopathic effects of essential oils of Pinus halepensis Miller: chemical composition and study of their antifungal and herbicidal activities. - Archives of Phytopathology and Plant Protection 48: 145-158.

[22] Heisey, R. M., Delwiche, C. (1983): A survey of California plants for water-extractable and volatile inhibitors. - Botanical Gazette 144: 382-390.

[23] Hussain, S. S., Mudasser, M., Sheikh, M. M., Manzoor, N. (2005): climate change and variability in mountain regions of Pakistan implications for water and agriculture. Pakistan Journal of Meteorology 2: 75-90.

[24] Hytonen, J. (1992): Allelopathic potential of peatland plant species on germination and early seedling growth of Scots pine, silver birch and downy birch. - Silva Fennica 6: 2431.

[25] Jobidon, R. (1986): Allelopathic potential of coniferous species to old-field weeds in eastern Quebec. - Forest Science 32: 112-118.

[26] Kato-Noguchi, H., Fushimi, H. Y., Shigemori, H. (2009): An allelopathic substance in red pine needles (Pinus densiflora). - Journal of Plant Physiology 166: 442-446.

[27] Kato-Noguchi, H., Fushimi, Y., Tanaka, Y., Teruya, T., Suenaga, K. (2011): Allelopathy of red pine: isolation and identification of an allelopathic substance in red pine needles. Plant Growth Regulation 65: 299-304.

[28] Khan, M. A., Hussain, I., Ahmad, E. (2008): Allelopathic effect of Eucalyptus (Eucalyptus camaldulensis L.) on germination and seedling growth of (Triticum aestivum L.). - Pakistan Journal of Weed Science Research 14: 9-18.

[29] Khosla, S., Singh, K., Sobti, S. (1981): Allelopathy of Pinus roxburghii Sargent. - Indian perfumer. - Journal of Integrative Medicine 5: 56-70.

[30] Kil, B. S. (1989): Allelopathic Effects of Five Pine Species in Korea. - In: Chou, C. H., Waller, G. R. (eds.) Phytochemical Ecology: Allelochemicals, Mycotoxins and Insect Pheromones and Allomones. Institute of Botany, Taipei, pp. 81-99.

[31] Kil, S. B., Yim, Y. J. (1983): Allelopathic effects of Pinus densiflora on undergrowth of red pine forest. - Journal of Chemical Ecology 9: 1135-1151.

[32] Kil, B. S., Kim, D. Y., Kim, Y. S., Lee, S. Y. (1991): Phytotoxic effects of naturally occurring chemicals from Pinus koraiensis on experimental species. - The Korean Journal of Ecology 14: 149-157.

[33] Kolesnichenko, M., Andryushchenko, G. (1978): Biochemical effect of some woody species on the Norway spruce. - Soviet Journal of Ecology 9: 325-328. 
[34] Lee, I. K., Monsi, M. (1963): Ecological studies on Pinus densiflora forest. Effects of plant substances on the floristic composition of the undergrowth. - Weed Management 76: 400-413.

[35] Madgil, D., Kapil, M. (1990): Allelopathic activity of Pinus roxburghii Sarg. and Rhododendron arboreum Sm. leaves. - Indian Forest 116: 512-514.

[36] Maestre, F. T., Cortina, J., Bautista, S., Bellot, J. (2003): Does Pinus halepensis facilitate the establishment of shrubs in Mediterranean semi-arid afforestations? - Forest Ecology and Management 176: 147-160.

[37] Melkania, N. P. (1984): Influence of leaf leachates of certain woody species on agricultural crops. - Indian Journal of Ecology 11: 82-86.

[38] Monnier, Y., Vila, B., Montes, N., Melou, A. B., Prevosto, B., Fernandez, C. (2011): Fertilization and allelopathy modify Pinus halepensis saplings crown acclimation to shade. - Trees 25: 497-507.

[39] Navarro-Cano, J. A., Barbera, G. G., Ruiz-Navarro, A., Castillo, V. M. (2009): Pine plantation bands limit seedling recruitment of a perennial grass under semiarid conditions. - Journal of Arid Environment 73: 120-126.

[40] Nekonam, M. S., Kraimmojeni, H., Sharifnabi, B., Razmjoo, J., Amini, H., Bahrami, F. (2014): Assessment of some medicinal plants for their allelopathic potential against redroot pigweed (Amaranthus retroflexus). - Journal of Plant Protection Research 54: 9095 .

[41] Nektarios, P. A., Economou, G., Avgoulas, C. (2005): Allelopathic effects of Pinus halepensis needles on turf grasses and biosensor plants. - Hortscienc 40: 246-250.

[42] Node, M., Yokotani, K. T., Suzuki, T., Kosemura, S., Hirata, H., Hirata, K., Hasegawa, K. (2003): Allelopathy of pinecone in Japanese red pine tree (Pinus densiflora Sieb. et Zucc.). - Weed Biology and Management 3: 111-116.

[43] Raana, G., Khalid, S., Qureshi, R., Sadia, S., Bajwa, A. A. (2012): Screening and evaluation of Euphorbia pulcherrima for weed management. - Pakistan Journal of Weed Science Research 18: 529-539.

[44] Refifa, T., Chahdoura, H., Ahmed, G. F., Helal, N. (2016): Allelopathic potential of Pinus halepensis needles. - Allelopathy Journal 38: 193-214.

[45] Rice, E. L. (1984): Allelopathy, $2^{\text {nd }}$ ed. - Academic Press, Orlando, FL.

[46] Robert, J. (1986): Allelopathic potential of coniferous species to old-field weeds in Eastern Quebec. - Forest Science 32: 112-118.

[47] Sahu, A., Devkota, A. (2013): Allelopathic effects of aqueous extract of leaves of Mikania micrantha H. B. K. on seed germination and seedling growth of Oryza sativa L. and Raphanus sativus L. - Science World Journal 11: 90-93.

[48] Satyal, P., Paudel, P., Raut, J., Deo, A., Dosoky, N. S., Setzer, W. N. (2013): Volatile constituents of Pinus roxburghii from Nepal. - Pharmacognosy Research 5: 43-50.

[49] Silva, J. A. T., Karimi, J., Mohsenzadeh, S., Dobranszki, J. (2015): Allelopathic potential of select gymnospermous trees. - Journal of Forest and Environmental Science 31(2): 109-118.

[50] Singh, H. P., Kohli, R. K., Batish, D. R. (2001): Allelopathic interference of Populus deltoides with some winter season crops. - Agronomy 21: 139-146.

[51] Singh, H. P., Batish, D. R., Kaur, S., Arora, K., kohli, R. K. (2006): $\alpha$-Pinene inhibits growth and induces oxidative stress in roots. - Annals of Botany 98: 1261-1269.

[52] Terzi, I., Kocacaliskan, I., Demir, Y. (2013): Allelopathic effects of some tree leaf extracts on seed germination and seedling growth of turf grasses. - Journal of Environmental Protection and Ecology 14: 1236-1243.

[53] Valera-Burgos, J., Diaz-Barradas, M. C., Zunzunegui, M. (2012): Effects of Pinus pinea litter on seed germination and seedling performance of three Mediterranean shrub species. - Plant Growth Regulation 66: 285-292. 
[54] Wang, R., Shaolin, P., Rensen, Z., Zengfu, X. U. (2009): Cloning, expression and wounding induction of $\beta$-caryophyllene synthase gene from Mikania micrantha HBK and allelopathic potential of $\beta$-caryophyllene. - Allelopathy Journal 24: 35-44.

[55] Williams, R. D., Hoagland, R. E. (1982): The effects of naturally occurring phenolic compounds on seed germination. - Weed Science 30: 206-212.

[56] Zafar, I., Fatima, A., Khan, S. J., Rehman, Z., Mehmud, S. (2010): GC-MS studies of needles essential oil of Pinus roxburghii and their antimicrobial activity from Pakistan. Electronic Journal of Environmental, Agricultural and Food Chemistry 9: 468-473.

[57] Zhou, Y. H., Yu, J. Q., Huang, L. F., Nogues, S. (2004): The relationship between $\mathrm{CO}_{2}$ assimilation, photosynthetic electron transport and water-water cycle in chill-exposed cucumber leaves under low light and subsequent recovery. - Plant, Cell \& Environment 27: 1503-1523. 